\title{
Gauges and functional measures in quantum gravity II: higher-derivative gravity
}

\author{
N. Ohta ${ }^{1, b}$, R. Percacci ${ }^{2,3, c}$, A. D. Pereira ${ }^{4, d}$ \\ ${ }^{1}$ Department of Physics, Kindai University, Higashi-Osaka, Osaka 577-8502, Japan \\ ${ }^{2}$ International School for Advanced Studies, via Bonomea 265, 34136 Trieste, Italy \\ ${ }^{3}$ INFN, Sezione di Trieste, Trieste, Italy \\ ${ }^{4}$ Departamento de Física Teórica, UERJ-Universidade do Estado do Rio de Janeiro, Rua São Francisco Xavier 524, Maracanã, Rio de Janeiro \\ 20550-013, Brazil
}

Received: 3 January 2017 / Accepted: 30 August 2017 / Published online: 15 September 2017

(C) The Author(s) 2017. This article is an open access publication

\begin{abstract}
We compute the one-loop divergences in a higher-derivative theory of gravity including Ricci tensor squared and Ricci scalar squared terms, in addition to the Hilbert and cosmological terms, on an (generally off-shell) Einstein background. We work with a two-parameter family of parametrizations of the graviton field, and a two-parameter family of gauges. We find that there are some choices of gauge or parametrization that reduce the dependence on the remaining parameters. The results are invariant under a recently discovered "duality" that involves the replacement of the densitized metric by a densitized inverse metric as the fundamental quantum variable.
\end{abstract}

\section{Introduction}

In a previous paper [1], hereafter referred to as I, we have examined the properties of quantum General Relativity (GR - the theory containing only terms up to second derivatives in the action) in a general four-parameter family of gauges and parametrizations. In this paper, we would like to extend the analysis to higher-derivative gravity (HDG).

By HDG, we will always mean the theory of gravity based on the metric as fundamental variable and an action that contains up to four derivatives. ${ }^{1}$ This theory is important because in four dimensions it is power-counting renormalizable [5] and asymptotically free [6-10]. In spite of these appealing

\footnotetext{
$\overline{1}$ There has been recently some progress on theories that contain more than four, and possibly infinitely many derivatives [2-4]. These also deserve the name HDG, but we shall not consider them here.

be-mail: ohtan@phys.kindai.ac.jp

c e-mail: percacci@sissa.it

de-mail: duarte763@gmail.com
}

properties, it has never been accepted as a viable fundamental theory for gravity, because of the presence of ghosts in perturbation theory. Over time, there have been many proposals trying to circumvent this problem. Among these let us mention here the following possibilities:

- The mass of the ghost is not a fixed parameter but is rather subject to strong (quadratic) running above the Planck threshold. Then the equation for the pole mass $m_{\text {phys }}^{2}=$ $m^{2}\left(k=m_{\text {phys }}\right)$ (where $m(k)$ is the running mass) may not have a solution $[6,11,12]$.

- The ghost may be an artifact of expanding around the wrong vacuum. The true vacuum of quadratic gravity (in the presence also of a Hilbert term) is not flat space but rather a kind of wave with wavelength of the order of the Planck length [13].

- The quadratic term is one of an infinite series and the sum of the series is a function that has no massive ghost pole. The ghost pole is an artifact of Taylor expanding this function to second order (see the aforementioned papers on non-local gravity and also [14]).

For further proposals see also [15-19]. So far, none of these arguments has convinced the community at large, so the issue of the ghosts remains open for the time being. However, the hope that a way out may exist has generated new interest in these theories in recent times, also among particle physicists [20-22]. It seems therefore appropriate to keep investigating the quantum properties of these theories.

In this paper we will extend previous results in several directions. Due to the complicated structure of the theory, calculations of one-loop divergences in HDG have usually been performed with a special four-derivative gauge-fixing term such that the four-derivative part of the Hessian is pro- 
portional to the square of the Laplacian. In this paper we will calculate the off-shell gauge dependence of the oneloop divergences by using the more conventional secondderivative gauge-fixing term that is commonly used in quantum GR, depending on two parameters $a$ and $b$, or a fourderivative variant of the same gauge fixing, containing an extra power of a Laplacian.

As another generalization, we assume that the quantum field is not the metric but the densitized metric

$\gamma_{\mu \nu}=g_{\mu \nu}\left(\sqrt{\operatorname{det} g_{\mu \nu}}\right)^{w}$,

or the densitized inverse metric

$\gamma^{\mu \nu}=g^{\mu \nu}\left(\sqrt{\operatorname{det} g^{\mu \nu}}\right)^{-w}=g^{\mu \nu}\left(\sqrt{\operatorname{det} g_{\mu \nu}}\right)^{w}$,

with weight $w$. Furthermore we allow the quantum theory to depend on another parameter $\omega$, which interpolates continuously between the linear background-field expansion (for $\omega=0$ )

$\gamma_{\mu \nu}=\bar{\gamma}_{\mu \nu}+\hat{h}_{\mu \nu}$

the exponential background-field expansions (for $\omega=1 / 2$ )

$\gamma_{\mu \nu}=\bar{\gamma}_{\mu \rho}\left(e^{\hat{h}}\right)^{\rho}{ }_{\nu} \quad$ or $\quad \gamma^{\mu \nu}=\left(e^{-\hat{h}}\right)^{\mu}{ }_{\rho} \bar{\gamma}^{\rho v}$,

and the linear expansion around the inverse densitized metric (for $\omega=1$ )

$\gamma^{\mu \nu}=\bar{\gamma}^{\mu \nu}-\hat{h}^{\mu \nu}$.

The density $\hat{h}_{\mu \nu}$ is then reexpressed as

$\hat{h}_{\mu \nu}=(\sqrt{\operatorname{det} \bar{g}})^{w} h_{\mu \nu} \quad$ or $\quad \hat{h}^{\mu \nu}=(\sqrt{\operatorname{det} \bar{g}})^{w} h^{\mu \nu}$

respectively, where $\bar{g}_{\mu \nu}$ is a background metric, related to the background density $\bar{\gamma}_{\mu \nu}$ as in (1.1) and (1.2). See also Eq. (2.14) below. The field $h_{\mu \nu}$ is used as integration variable in the functional integral. The properties of quantum GR in this general four-parameter family of gauges and parametrizations have been investigated in an accompanying paper [1].

Here we extend the analysis of I to HDG. We now have four independent couplings instead of two, so the expressions for the divergences are in general much more complicated than in I and so is the interpretation of the results. The expressions simplify somewhat, and they are reported explicitly, in two limits: the "four derivative gravity (4DG) limit" in which the Einstein-Hilbert terms can be neglected relative to the curvature squared terms, and the "Einstein-Hilbert (EH) limit" where the opposite holds. In the EH limit, the action is the same as the one considered in I, but the analysis that we perform here differs in two respects: first, we choose a more general Einstein background, instead of the maximally symmetric background of I. This allows us to discriminate two divergent terms quadratic in curvature, rather than a single one as in I. Secondly, the cutoff in each spin sector is chosen to depend on the corresponding Lichnerowicz Laplacian, rather than the Bochner Laplacian $-\nabla^{2}$ as in I. Thus, comparison with I yields some information on the cutoff dependence of these non-universal results. Still, we find that in this limit the qualitative picture is the same. Similar calculations of divergent terms with different parametrizations in four dimensions have been given in [23].

In four dimensions and in the 4DG-limit, all divergences are universal, i.e. independent of both gauge and parametrization. In particular, the logarithmic divergences are related to the well-known universal beta functions of HDG $[9,10]$. As in I, we find that all divergences are invariant under a "duality" transformation that consists in the replacement

$\begin{aligned} \omega & \rightarrow 1-\omega, \\ w & \rightarrow w+\frac{4}{d} .\end{aligned}$

Let us discuss a little more the role of the functional measure in these considerations. In I we have used the word "measure" synonymously with "choice of quantum field in the functional integral" but we have left the definition of the functional measure a bit implicit. The reason for this is that the Wilsonian cutoff $k$ that we have used to calculate the divergences does not regulate the divergences in the functional measure, if there are any, and so the results would have been independent of this choice anyway. The consistent interpretation of the results of $\mathrm{I}$ is that they give the correct divergences of the functional integrals when the measure is given by

$\Pi_{x} d h_{\mu v}(x)$,

where $h_{\mu \nu}$ is the purely tensorial quantum field defined as in (2.14), independently of $m$ and $\omega$.

Since the functional measure was always kept fixed, the dependence of the results on the parameters $m$ and $\omega$ resulted entirely from the different forms of the Hessians, which in turn was due to the different forms of the expansion of the action (see Eqs. (2.13) and (2.15)).

If one decided to use the same expansion but a different ultralocal measure, for example

$\Pi_{x} d \hat{h}_{\mu \nu}(x)=\Pi_{x}(\operatorname{det} \bar{g}(x))^{w / 2} d h_{\mu \nu}(x)$,

then the divergences would differ by terms of the form $\delta(0)$ times the volume. Such terms would affect the power-law divergences coefficients. 
We conclude this introduction by listing the contents of the following sections. Section 2 contains details of our calculations of one-loop divergences. In Sect. 3 we give a formal proof (at the level of determinants) that the on-shell effective action in $d=4$ is gauge independent. (In particular, the effective action of HDG in the 4DG-limit on an Einstein space is gauge-independent.) The main results are presented in Sect. 4. In Sect. 5, we discuss how the results can be obtained for $d=4$ conformal gravity. In Sect. 6, we point out that our results show that the duality found in our previous paper I is valid in HDG as well. Section 5 contains a discussion and conclusions. In the appendix, we summarize the heat kernel coefficients for Lichnerowicz Laplacians on an Einstein manifold.

\section{The one-loop effective action}

\subsection{The HDG actions and their equations of motion}

In this paper we will consider actions of the general form

$S(g)=\int d^{d} x \sqrt{\mp g}\left[ \pm Z_{N}(R-2 \Lambda)+\alpha R^{2}+\beta R_{\mu \nu}^{2}\right]$,

where $Z_{N}=1 /(16 \pi G), \Lambda$ is the cosmological constant, $\alpha$, $\beta$ are the higher-derivative couplings. The upper sign refers to the Minkowski signature, the lower one to the Euclidean signature. This is not the most general HDG action, because we omit a term $\gamma R_{\mu \nu \rho \sigma}^{2}$; however, in $d \leq 4$ this is not a very strong restriction, because the "Gauss-Bonnet" or "Euler" combination

$G=R_{\mu \nu \alpha \beta}^{2}-4 R_{\mu \nu}^{2}+R^{2}$,

is either zero (in $d=2,3$ ) or a total derivative (in $d=4$ ). Using the identity for the Weyl tensor $C_{\mu \nu \rho \sigma}$ :

$$
\begin{aligned}
C^{2} & \equiv C_{\mu \nu \rho \sigma} C^{\mu \nu \rho \sigma} \\
& =R_{\mu \nu \alpha \beta}^{2}-\frac{4}{d-2} R_{\mu \nu}^{2}+\frac{2}{(d-1)(d-2)} R^{2},
\end{aligned}
$$

one has

$C^{2}=G+\frac{2(d-3)}{d-2} W ; \quad W=2 R_{\mu \nu}^{2}-\frac{d}{2(d-1)} R^{2}$.

Thus, modulo terms proportional to $G$, we can replace $\alpha R^{2}+$ $\beta R_{\mu \nu}^{2}$ by

$$
\frac{1}{2 \lambda} W+\frac{1}{\xi} R^{2}
$$

The integral of $W$, like the integral of $C^{2}$, is Weyl-invariant in $d=4$.
The equations of motion are

$$
\pm Z_{N}\left(G_{\mu \nu}+\Lambda g_{\mu \nu}\right)+\alpha E_{\mu \nu}^{(1)}+\beta E_{\mu \nu}^{(2)}=0
$$

where $G_{\mu \nu}$ is the Einstein tensor and

$$
\begin{aligned}
E_{\mu \nu}^{(1)}= & 2 R R_{\mu \nu}-2 \nabla_{\mu} \nabla_{\nu} R+g_{\mu \nu}\left(2 \square R-\frac{1}{2} R^{2}\right), \\
E_{\mu \nu}^{(2)}= & 2 R_{\mu \lambda} R_{\nu}^{\lambda}-2 \nabla^{\lambda} \nabla_{(\mu} R_{\nu) \lambda}+\square R_{\mu \nu} \\
& +\frac{1}{2}\left(\square R-R_{\lambda \rho}^{2}\right) g_{\mu \nu} .
\end{aligned}
$$

Let us observe that in the case $Z_{N}=0$ and $d=4$ the Einstein condition

$$
R_{\mu \nu}=\frac{1}{d} R g_{\mu \nu}
$$

is enough to fulfill the equations of motion. The scalar curvature remains an undetermined constant, since the action is scale-invariant. On the other hand when $Z_{N} \neq 0$ the equations of motion together with the Einstein condition further require

$\mathcal{P} \equiv \frac{4-d}{2}\left(\alpha+\frac{\beta}{d}\right) R^{2} \mp \frac{d-2}{2} Z_{N}\left(R-\frac{2 d}{d-2} \Lambda\right)=0$.

In particular, in four dimensions the higher-derivative terms do not contribute and the equation of motion is the same as the trace of the Einstein equations with cosmological constant:

$E \equiv R-\frac{2 d}{d-2} \Lambda=0$

To summarize, the choice of the Einstein condition means that the background is "almost on shell" in the sense that all the equations of motion are satisfied except, in general, for the trace equation (2.9). In the special case of pure HDG (no Einstein-Hilbert term) in $d=4$ the background is completely on-shell.

\subsection{Quadratic expansion}

The action has to be thought of as a functional of the quantum field $\gamma_{\mu \nu}$ or $\gamma^{\mu \nu}$ defined as in (1.1) or (1.2). For $w \neq-1 / d$ these relations can be inverted to yield

$g_{\mu \nu}=\gamma_{\mu \nu}\left(\operatorname{det}\left(\gamma_{\mu \nu}\right)\right)^{m} ; \quad g^{\mu \nu}=\gamma^{\mu \nu}\left(\operatorname{det}\left(\gamma_{\mu \nu}\right)\right)^{-m}$,

where

$$
\frac{w}{2}=-\frac{m}{1+d m} \quad \text { or } \quad \frac{w}{2}=\frac{m}{1+d m},
$$


respectively. Conversely, $m=-\frac{w / 2}{1+d w / 2}$ for (1.1) and $m=$ $\frac{w / 2}{1-d w / 2}$ for (1.2). We observe that the relation between $m$ and $w / 2$ is an involution. We choose to treat $m$ as an independent free parameter. All dependence on $m$ can be translated into a dependence on $w$ if needed, using the preceding formulas.

The quantum field is then expanded as in (1.3) or (1.4) or (1.5), and for the calculation of the one-loop divergences we need the terms to second order in the fluctuation. As explained in [1], we can start from the quadratic expansion of the action in $\delta g_{\mu \nu}$, which has been given in detail in [24], and then use

$\delta g_{\mu \nu}=\delta g_{\mu \nu}^{(1)}+\delta g_{\mu \nu}^{(2)}+\cdots$,

where $\delta g_{\mu \nu}^{(n)}$ contains $n$ powers of the tensor fluctuation

$h_{\mu \nu}=(\operatorname{det} \bar{\gamma})^{m} \hat{h}_{\mu \nu}$.

For all four types of expansion considered here, we have

$$
\begin{aligned}
\delta g_{\mu \nu}^{(1)}= & h_{\mu \nu}+m \bar{g}_{\mu \nu} h, \\
\delta g_{\mu \nu}^{(2)}= & \omega h_{\mu \rho} h^{\rho}{ }_{\nu}+m h h_{\mu \nu}+m\left(\omega-\frac{1}{2}\right) \bar{g}_{\mu \nu} h^{\alpha \beta} h_{\alpha \beta} \\
& +\frac{1}{2} m^{2} \bar{g}_{\mu \nu} h^{2} .
\end{aligned}
$$

The choice $\omega=0$ corresponds to the linear expansion of metric (1.3), $\omega=1 / 2$ corresponds to the exponential expansion (1.4) (and it does not matter if one starts from the metric or from the inverse metric) and $\omega=1$ corresponds to the linear expansion of the inverse metric (1.5).

In the following we will use these expansions in the action (2.1). We note that, for given $g_{\mu \nu}$ and $\bar{g}_{\mu \nu}$, different values of $\omega$ and $m$ will give different fluctuation fields $h_{\mu \nu}$ and conversely for given $h_{\mu \nu}$ and $\bar{g}_{\mu \nu}$, different values of $\omega$ and $m$ will give different total metrics $g_{\mu \nu}$. In the following calculations the action (2.1), as a functional of the total metric, will always be kept fixed and also the functional measure for the quantum field will be kept fixed.

\subsection{Lichnerowicz Laplacians}

The evaluation of the one-loop divergences is based on the knowledge of the coefficients of the small-time expansion of the heat kernel of the kinetic operator appearing in the gauge-fixed Hessian. We will denote $\bar{\nabla}$ the covariant derivative defined by the background metric $\bar{g}, \square=\bar{\nabla}^{2}$ the d'Alembertian and $-\bar{\nabla}^{2}$ its Euclidean analog, known as Bochner Laplacian. In GR, the expansion of the action generates non-minimal terms that can be eliminated by choosing the de Donder gauge. The rest can be written in terms of Laplacians. Similarly, the second variation of the HDG action contains non-Laplacian terms such as $\bar{\nabla}_{\mu} \bar{\nabla}_{\nu} \bar{\nabla}_{\rho} \bar{\nabla}_{\sigma}$, $\square \bar{\nabla}_{\mu} \bar{\nabla}_{\nu} \bar{g}_{\rho \sigma}+\bar{g}_{\mu \nu} \bar{\nabla}_{\rho} \bar{\nabla}_{\sigma} \square$ and $\bar{g}_{\nu \sigma} \bar{\nabla}_{\mu} \square \bar{\nabla}_{\rho}+\bar{g}_{\mu \rho} \bar{\nabla}_{\nu} \square \bar{\nabla}_{\sigma}$, acting on $h_{\rho \sigma}$. The heat kernel expansion for such operators is not known, so we will use the York decomposition to rewrite the Hessian as minimal operators acting on fields with definite spin. For this purpose, it is essential to rewrite all occurrences of $\square$ in terms of the Lichnerowicz Laplacians acting on spin- 0 , spin- 1 and spin- 2 fields, which are defined (in Euclidean signature) as follows:

$$
\begin{aligned}
\Delta_{L 0} \phi= & -\bar{\nabla}^{2} \phi, \\
\Delta_{L 1} A_{\mu}= & -\bar{\nabla}^{2} A_{\mu}+\bar{R}_{\mu}{ }^{\rho} A_{\rho}, \\
\Delta_{L 2} h_{\mu \nu}= & -\bar{\nabla}^{2} h_{\mu \nu}+\bar{R}_{\mu}{ }^{\rho} h_{\rho \nu}+\bar{R}_{\nu}{ }^{\rho} h_{\mu \rho}-\bar{R}_{\mu \rho v \sigma} h^{\rho \sigma} \\
& -\bar{R}_{\mu \rho v \sigma} h^{\sigma \rho} .
\end{aligned}
$$

These operators have the following useful properties:

$$
\begin{aligned}
& \Delta_{L 1} \bar{\nabla}_{\mu} \phi=\bar{\nabla}_{\mu} \Delta_{L 0} \phi, \\
& \bar{\nabla}_{\mu} \Delta_{L 1} \xi^{\mu}=\Delta_{L 0} \bar{\nabla}_{\mu} \xi^{\mu}, \\
& \Delta_{L 2}\left(\bar{\nabla}_{\mu} \bar{\nabla}_{\nu} \phi\right)=\bar{\nabla}_{\mu} \bar{\nabla}_{\nu} \Delta_{L 0} \phi, \\
& \Delta_{L 2}\left(\bar{\nabla}_{\mu} \xi_{\nu}+\bar{\nabla}_{\nu} \xi_{\mu}\right)=\bar{\nabla}_{\mu} \Delta_{L 1} \xi_{\nu}+\bar{\nabla}_{\nu} \Delta_{L 1} \xi_{\mu}, \\
& \Delta_{L 2} \bar{g}_{\mu \nu} \phi=\bar{g}_{\mu \nu} \Delta_{L 0} \phi .
\end{aligned}
$$

The York decomposition leads to significant simplifications when the background metric is an Einstein space, i.e. satisfies the condition (2.8). (As discussed in Sect. 2.1, this is not enough to put the background on-shell.) In the following we will always assume that the background is Einstein, but not that it satisfies (2.10).

Now consider the second variations of the curvature squared terms in the action, as given in eqs. (B.6) and (B.7) in [24]. The terms containing the Riemann tensor can be combined with certain terms containing $\square$ to give Lichnerowicz Laplacians. Further using (2.8), one arrives at

$$
\begin{aligned}
\alpha \delta & g^{\mu \nu}\left[\bar{\nabla}_{\mu} \bar{\nabla}_{\nu} \bar{\nabla}_{\alpha} \bar{\nabla}_{\beta}-2 \bar{g}_{\mu \nu} \square \bar{\nabla}_{\alpha} \bar{\nabla}_{\beta}+\bar{g}_{\mu \nu} \bar{g}_{\alpha \beta} \square^{2}\right. \\
& -\bar{g}_{\nu \beta} \bar{R} \bar{\nabla}_{\mu} \bar{\nabla}_{\alpha}+\frac{d-2}{2} \bar{R} \bar{g}_{\mu \nu} \bar{\nabla}_{\alpha} \bar{\nabla}_{\beta}+\frac{4-d}{2 d} \bar{g}_{\mu \nu} \bar{g}_{\alpha \beta} \bar{R} \square \\
& -\frac{1}{2} \bar{g}_{\mu \alpha} \bar{g}_{\nu \beta} \bar{R} \Delta_{L 2}+\left(\frac{1}{d^{2}}-\frac{1}{d}+\frac{1}{8}\right) \bar{R}^{2} \bar{g}_{\mu \nu} \bar{g}_{\alpha \beta} \\
& \left.+\left(\frac{2}{d}-\frac{1}{4}\right) \bar{R}^{2} \bar{g}_{\mu \alpha} \bar{g}_{\nu \beta}\right] \delta g^{\alpha \beta}
\end{aligned}
$$

and

$$
\begin{aligned}
& \beta \delta g^{\mu \nu}\left[\frac{1}{2} \bar{\nabla}_{\mu} \bar{\nabla}_{\nu} \bar{\nabla}_{\alpha} \bar{\nabla}_{\beta}-\frac{1}{2} \bar{g}_{\mu \nu} \square \bar{\nabla}_{\alpha} \bar{\nabla}_{\beta}-\frac{1}{2} \bar{g}_{\nu \beta} \bar{\nabla}_{\mu} \square \bar{\nabla}_{\alpha}\right. \\
& +\frac{1}{4} \bar{g}_{\mu \nu} \bar{g}_{\alpha \beta} \square^{2}+\frac{1}{4} \bar{g}_{\mu \alpha} \bar{g}_{\nu \beta} \Delta_{L 2}\left(\Delta_{L 2}-\frac{6}{d} \bar{R}\right) \\
& -\frac{3}{2 d} \bar{R}_{{ }_{\nu \beta}} \bar{\nabla}_{\mu} \bar{\nabla}_{\alpha}
\end{aligned}
$$




$$
\begin{aligned}
& +\frac{1}{d} \bar{R} \bar{g}_{\mu \nu} \bar{\nabla}_{\alpha} \bar{\nabla}_{\beta}-\frac{1}{4 d} \bar{R} \bar{g}_{\mu \nu} \bar{g}_{\alpha \beta} \square \\
& \left.+\frac{12-d}{4 d^{2}} \bar{R}^{2} \bar{g}_{\mu \alpha} \bar{g}_{\nu \beta}+\frac{d-8}{8 d^{2}} \bar{R}^{2} \bar{g}_{\mu \nu} \bar{g}_{\alpha \beta}\right] \delta g^{\alpha \beta} .
\end{aligned}
$$

All tensor structures are provided by the background metric. We note that this procedure of eliminating the Riemann tensor does not work in the case of the second variation of the Riemann squared term, which is the reason why we do not consider such a term.

\subsection{York decomposition}

The York decomposition is defined by

$$
\begin{aligned}
h_{\mu \nu}= & h_{\mu \nu}^{T T}+\bar{\nabla}_{\mu} \xi_{\nu}+\bar{\nabla}_{\nu} \xi_{\mu}+\bar{\nabla}_{\mu} \bar{\nabla}_{\nu} \sigma-\frac{1}{d} \bar{g}_{\mu \nu} \bar{\nabla}^{2} \sigma \\
& +\frac{1}{d} \bar{g}_{\mu \nu} h,
\end{aligned}
$$

where $h_{\mu \nu}^{T T}$ is transverse and tracefree, and $\hat{\xi}_{\mu}$ is transverse. We will use

$\hat{\xi}_{\mu}=\sqrt{-\bar{\nabla}^{2}-\frac{\bar{R}}{d}} \xi_{\mu} ; \quad \hat{\sigma}=\sqrt{-\bar{\nabla}^{2}} \sqrt{-\bar{\nabla}^{2}-\frac{\bar{R}}{d-1}} \sigma$.

Employing (2.24) and (2.25) and assuming that the background is an Einstein space, one finds

$$
\begin{aligned}
& \int d^{d} x \sqrt{\bar{g}} h_{\mu \nu} h^{\mu \nu} \\
& =\int d^{d} x \sqrt{\bar{g}}\left[h_{\mu \nu}^{T T} h^{T T \mu \nu}+2 \hat{\xi}_{\mu} \hat{\xi}^{\mu}+\frac{d-1}{d} \hat{\sigma}^{2}+\frac{1}{d} h^{2}\right] .
\end{aligned}
$$

Using the properties (2.17)-(2.21), we have

$$
\begin{aligned}
& \int d^{d} x \sqrt{\bar{g}} h_{\mu \nu} \Delta_{L 2} h^{\mu \nu} \\
& =\int d^{d} x \sqrt{\bar{g}}\left[h_{\mu \nu}^{T T} \Delta_{L 2} h^{T T \mu \nu}+2 \hat{\xi}_{\mu} \Delta_{L 1} \hat{\xi}^{\mu}\right. \\
& \left.\quad+\frac{d-1}{d} \hat{\sigma} \Delta_{L 0} \hat{\sigma}+\frac{1}{d} h \Delta_{L 0} h\right], \\
& \int d^{d} x \sqrt{\bar{g}} h_{\mu \nu}\left(\Delta_{L 2}\right)^{2} h^{\mu \nu} \\
& =\int d^{d} x \sqrt{\bar{g}}\left[h_{\mu \nu}^{T T}\left(\Delta_{L 2}\right)^{2} h^{T T \mu \nu}+2 \hat{\xi}_{\mu}\left(\Delta_{L 1}\right)^{2} \hat{\xi}^{\mu}\right. \\
& \left.\quad+\frac{d-1}{d} \hat{\sigma}\left(\Delta_{L 0}\right)^{2} \hat{\sigma}+\frac{1}{d} h\left(\Delta_{L 0}\right)^{2} h\right],
\end{aligned}
$$

and so on.
Furthermore using

$$
\begin{aligned}
\bar{\nabla}_{\alpha} h^{\alpha}{ }_{\nu}= & \left(\bar{\nabla}^{2}+\frac{\bar{R}}{d}\right) \xi_{v}+\frac{d-1}{d} \bar{\nabla}_{\nu}\left(\bar{\nabla}^{2}+\frac{\bar{R}}{d-1}\right) \sigma \\
& +\frac{1}{d} \bar{\nabla}_{\nu} h
\end{aligned}
$$

and

$\bar{\nabla}_{\alpha} \bar{\nabla}_{\beta} h^{\alpha \beta}=\frac{d-1}{d} \bar{\nabla}^{2}\left(\bar{\nabla}^{2}+\frac{\bar{R}}{d-1}\right) \sigma+\frac{1}{d} \bar{\nabla}^{2} h$,

we find that

$$
\begin{aligned}
& \int d^{d} x \sqrt{\bar{g}} h^{\mu \nu} \bar{\nabla}_{\mu} \bar{\nabla}_{\nu} \bar{\nabla}_{\alpha} \bar{\nabla}_{\beta} h^{\alpha \beta} \\
& =\int d^{d} x \sqrt{\bar{g}}\left[\left(\frac{d-1}{d}\right)^{2} \sigma \square^{2}\left(\square+\frac{\bar{R}}{d-1}\right) \sigma\right. \\
& \left.\quad+2 \frac{d-1}{d^{2}} h \square^{2}\left(\square+\frac{\bar{R}}{d-1}\right) \sigma+\frac{1}{d^{2}} h \square^{2} h\right], \quad \text { (2.31) } \\
& \int d^{d} x \sqrt{\bar{g}} h^{\mu \nu} \bar{\nabla}_{\mu} \bar{\nabla}_{\alpha} h^{\alpha}{ }_{\nu}=\int d^{d} x \sqrt{\bar{g}} \\
& \quad \times\left[-\xi_{\mu}\left(\square+\frac{\bar{R}}{d}\right)^{2} \xi^{\mu}+\left(\frac{d-1}{d}\right)^{2} \sigma \square\left(\square+\frac{\bar{R}}{d-1}\right)^{2} \sigma\right. \\
& \left.\quad+2 \frac{d-1}{d^{2}} h \square\left(\square+\frac{\bar{R}}{d-1}\right) \sigma+\frac{1}{d^{2}} h \square h\right], \\
& \int d^{d} x \sqrt{\bar{g}} h^{\mu \nu} \bar{\nabla}_{\mu} \square \bar{\nabla}_{\alpha} h^{\alpha}{ }_{\nu} \\
& =\int d^{d} x \sqrt{\bar{g}}\left[-\xi_{\mu} \square\left(\square+\frac{\bar{R}}{d}\right)^{2} \xi^{\mu}\right. \\
& \quad+\left(\frac{d-1}{d}\right)^{2} \sigma \square\left(\square+\frac{\bar{R}}{d}\right)\left(\square+\frac{\bar{R}}{d-1}\right)^{2} \sigma \\
& \quad+2 \frac{d-1}{d^{2}} h \square\left(\square+\frac{\bar{R}}{d}\right)\left(\square+\frac{\bar{R}}{d-1}\right) \sigma \\
& \left.+\frac{1}{d^{2}} h \square\left(\square+\frac{\bar{R}}{d}\right) h\right] .
\end{aligned}
$$

\subsection{The decomposed Hessian}

The expansion of the Euclidean action in powers of $h$ has the following quadratic part:

$$
\begin{aligned}
S^{(2)}= & \int d^{d} x \sqrt{\bar{g}}\left[h_{\mu \nu}^{T T} H^{T T} h^{T T \mu \nu}+\hat{\xi}_{\mu} H^{\xi \xi} \hat{\xi}^{\mu}+\hat{\sigma} H^{\sigma \sigma} \hat{\sigma}\right. \\
& \left.+\hat{\sigma} H^{\sigma h} h+h H^{h \sigma} \hat{\sigma}+h H^{h h} h\right],
\end{aligned}
$$

with

$$
\begin{aligned}
H^{T T}= & \frac{1}{4} Z_{N}\left(\Delta_{L 2}-\frac{2 \bar{R}}{d}+\frac{d-2}{d}(1-2 \omega)(1+d m) \bar{E}\right) \\
& +\frac{\beta}{4}\left(\left(\Delta_{L 2}\right)^{2}-\frac{6}{d} \bar{R} \Delta_{L 2}\right.
\end{aligned}
$$




$$
\begin{aligned}
& \left.+\frac{8-(d-4)(1-2 \omega)(1+d m)}{d^{2}} \bar{R}^{2}\right) \\
& -\frac{\alpha}{2} \bar{R}\left(\Delta_{L 2}-\frac{4-(d-4)(1-2 \omega)(1+d m)}{2 d} \bar{R}\right), \\
& H^{\xi \xi}=\frac{(1-2 \omega)(1+d m)}{2 d} \\
& \times\left[(d-2) Z_{N} \bar{E}-\frac{d-4}{d}(d \alpha+\beta) \bar{R}^{2}\right], \\
& H^{\sigma \sigma}=\frac{d-1}{d}\left\{-\frac{d-2}{4 d} Z_{N}\left(\Delta_{L 0}-(1-2 \omega)(1+d m) \bar{E}\right)\right. \\
& +\frac{d-1}{d} \alpha\left[\left(\Delta_{L_{0}}\right)^{2}+\frac{d-4}{2(d-1)}\right. \\
& \left.\times \bar{R}\left(\Delta_{L 0}-\frac{(1-2 \omega)(1+d m)}{2} \bar{R}\right)\right] \\
& +\frac{\beta}{4}\left[\left(\Delta_{L 0}\right)^{2}+\frac{2(d-4)}{d^{2}}\right. \\
& \left.\left.\times \bar{R}\left(\Delta_{L 0}-\frac{(1-2 \omega)(1+d m)}{2} \bar{R}\right)\right]\right\}, \\
& H^{\sigma h}=H^{h \sigma}=\frac{d-1}{2 d}(1+d m) \sqrt{\Delta_{L 0}} \\
& \times \sqrt{\Delta_{L 0}-\frac{\bar{R}}{d-1}}\left[-Z_{N} \frac{d-2}{2 d}\right. \\
& +\alpha \frac{2(d-1)}{d}\left(\Delta_{L 0}+\frac{d-4}{2(d-1)} \bar{R}\right) \\
& \left.+\frac{\beta}{2}\left(\Delta_{L 0}+\frac{2(d-4)}{d^{2}} \bar{R}\right)\right] \text {, } \\
& H^{h h}=\frac{d-1}{d}(1+d m)^{2} \\
& \times\left\{-Z_{N} \frac{d-2}{4 d}\left(\Delta_{L 0}-\frac{\bar{R}}{d-1}+\frac{d-2+d^{2} m+4 \omega}{2(d-1)(1+d m)} \bar{E}\right)\right. \\
& +\alpha \frac{d-1}{d}\left(\left(\Delta_{L 0}\right)^{2}+\frac{d-6}{2(d-1)} \bar{R} \Delta_{L 0}\right. \\
& \left.+\frac{(d-4)\left(d-6-4 d m+d^{2} m+4 \omega\right)}{8(d-1)^{2}(1+d m)} \bar{R}^{2}\right) \\
& +\frac{\beta}{4}\left(\left(\Delta_{L 0}\right)^{2}+\frac{d^{2}-10 d+8}{d^{2}(d-1)} \bar{R} \Delta_{L 0}\right. \\
& \left.\left.+\frac{(d-4)\left(d-6-4 d m+d^{2} m+4 \omega\right)}{2(d-1) d^{2}(1+d m)} \bar{R}^{2}\right)\right\},
\end{aligned}
$$

where $\bar{E}=\bar{R}-\frac{2 d \Lambda}{d-2}$.

If we define the scalar gauge-invariant degree of freedom
$s=\frac{\sqrt{\Delta_{L 0}}}{\sqrt{\Delta_{L 0}-\frac{\bar{R}}{d-1}}} \hat{\sigma}+(1+d m) h$,

the scalar sector of the Hessian can be rewritten as

$\int d^{d} x \sqrt{\bar{g}}\left[s H_{s}^{s s} s+s H_{s}^{s h} h+h H_{s}^{h s} s+h H_{s}^{h h} h\right]$,

where

$$
\begin{aligned}
H_{s}^{s s}= & \frac{d-1}{4 d^{3}} \frac{\Delta_{L 0}-\frac{\bar{R}}{d-1}}{\Delta_{L 0}}\left\{-d(d-2) Z_{N}\right. \\
& \times\left[\Delta_{L 0}-(1+d m)(1-2 \omega)\left(\bar{R}-\frac{2 d \Lambda}{d-2}\right)\right] \\
& +4 d(d-1) \alpha\left[\left(\Delta_{L 0}\right)^{2}+\frac{d-4}{2(d-1)} \Delta_{L 0} \bar{R}\right. \\
& \left.-\frac{d-4}{4(d-1)}(1+d m)(1-2 \omega) \bar{R}^{2}\right] \\
& +d^{2} \beta\left[\left(\Delta_{L 0}\right)^{2}+2 \frac{d-4}{d^{2}} \Delta_{L 0} \bar{R}\right. \\
& \left.\left.-\frac{d-4}{d^{2}}(1+d m)(1-2 \omega) \bar{R}^{2}\right]\right\}, \\
H_{s}^{s h}= & H_{s}^{h s}=-\frac{d-1}{2 d^{2}} \frac{\Delta_{L 0}-\frac{\bar{R}}{d-1}}{\Delta_{L 0}}(1+d m)^{2}(1-2 \omega) \overline{\mathcal{P}}, \\
H_{s}^{h h}= & -\frac{1}{4 d^{2}} \frac{(1+d m)}{\Delta_{L 0}}\left[d \left(2 d(d-1) m^{2}(2 \omega-1)\right.\right. \\
& +d m(8 \omega-3)-4 m(2 \omega-1)+4 \omega-1) \Delta_{L 0} \\
& \left.+2(1+d m)^{2}(1-2 \omega) \bar{R}\right] \overline{\mathcal{P}} .
\end{aligned}
$$

This form of the Hessian has the virtue that all the terms that contain $h$ are proportional to the equation of motion. This shows that the field $h$ can be completely disregarded on shell, as one would expect of a gauge-variant variable. This, however, depends on the choice of basis in the space of fields and is only true when the other scalar degree of freedom is gauge-invariant.

On the other hand, this form of the Hessian has the unpleasant feature that the kinetic operator of the field $s$ is non-local. One cannot generally absorb the non-local prefactor in a redefinition of $s$, because one is not allowed to perform non-local redefinitions of physical fields.

One notices, however, that the terms with the lowest power of $\Delta_{L 0}$ in each of the three lines in $H_{s}^{s s}$ is proportional to $(1+d m)(1-2 \omega)$. Therefore, if either $\omega=1 / 2$ (exponential parametrization) or $m=-1 / d$ (the "unimodular" measure), each of the square brackets in (2.42) is proportional to $\Delta_{L 0}$ and the Hessian of $s$ becomes local.

We further observe that for $\omega=1 / 2$ also the mixed term vanishes and the term $H_{s}^{h h}$ becomes local, whereas for 
$m=-1 / d$ all terms containing $h$ vanish, as expected in the unimodular theory.

Furthermore, $H_{s}^{s s}$ becomes local and independent of $m$ and $\omega$ for pure four-derivative gravity $\left(Z_{N}=0\right)$ when $d=4$. In this case the whole Hessian becomes just

$$
\begin{aligned}
& \frac{1}{4} \beta h_{\mu \nu}^{T T}\left(\Delta_{L 2}-\frac{\bar{R}}{2}\right)\left(\Delta_{L 2}-\left(1+\frac{2 \alpha}{\beta}\right) \bar{R}\right) h^{T T \mu \nu} \\
& +\frac{3}{16}(3 \alpha+\beta) s \Delta_{L 0}\left(\Delta_{L 0}-\frac{\bar{R}}{3}\right) s .
\end{aligned}
$$

Finally we observe that in the conformal case $d=4, \beta=$ $-3 \alpha$ (which corresponds to having only the term $W$ in the action) the $s s$ term in the Hessian vanishes too, leaving only the pure spin-2 degree of freedom, with Hessian

$$
\frac{1}{4} \beta h_{\mu \nu}^{T T}\left(\Delta_{L 2}-\frac{\bar{R}}{2}\right)\left(\Delta_{L 2}-\frac{\bar{R}}{3}\right) h^{T T \mu \nu} .
$$

\subsection{Two-derivative gauge-fixing terms}

We now turn to the discussion of the gauge-fixing and Faddeev-Popov (FP) ghost terms. In most of the following, we use the same two-derivative gauge-fixing term as in I [1]. The gauge-fixing function is defined as

$F_{\mu}=\bar{\nabla}_{\alpha} h_{\mu}^{\alpha}-\frac{\bar{b}+1}{d} \bar{\nabla}_{\mu} h$

with a gauge-fixing parameter $-\infty<\bar{b}<\infty$. Here $h_{\mu \nu}$ is the tensorial quantum field defined above and the bars on the covariant derivatives mean that they are calculated from the background metric $\bar{g}_{\mu \nu}$. The simplest way to derive the gauge-fixing and FP ghost terms is to use the BRST transformations, which are obtained by replacing the gauge parameters with the FP ghosts. In the present case, we have

$\delta_{B} g_{\mu \nu}=-\delta \lambda\left[g_{\rho \nu} \nabla_{\mu} C^{\rho}+g_{\rho \mu} \nabla_{\nu} C^{\rho}\right]$,

where $C^{\mu}$ is the FP ghost, and $\delta \lambda$ is an anticommuting parameter. The BRST transformation for other fields is derived by the requirement of the nilpotency of the transformation:

$\delta_{B} C^{\mu}=\delta \lambda C^{\rho} \partial_{\rho} C^{\mu}, \quad \delta_{B} \bar{C}_{\mu}=i \delta \lambda B_{\mu}, \quad \delta_{B} B_{\mu}=0$,

where $\bar{C}_{\mu}$ is the FP anti-ghost and $B_{\mu}$ is an auxiliary field which enforces the gauge-fixing condition.

In order to derive the gauge-fixing and FP terms, we have to know the transformations on the fluctuation field $h_{\mu \nu}$. From the first relation in Eq. (2.15), we have

$\delta_{B} h_{\mu \nu}+m \bar{g}_{\mu \nu} \delta_{B} h+\cdots=-\delta \lambda\left[g_{\rho \nu} \nabla_{\mu} C^{\rho}+g_{\rho \mu} \nabla_{\nu} C^{\rho}\right]$.

Anticipating that we will compute the effective action for vanishing expectation value of $h_{\mu \nu}$, we can restrict this to the linear terms to obtain

$\delta_{B} h_{\mu \nu}=-\delta \lambda\left[\bar{\nabla}_{\mu} C_{\nu}+\bar{\nabla}_{\nu} C_{\mu}-\frac{2 m}{1+d m} \bar{g}_{\mu \nu} \bar{\nabla}_{\rho} C^{\rho}\right]$.

The gauge-fixing and FP terms are then given as [25,26]

$$
\begin{aligned}
\mathcal{L}_{\mathrm{GF}+\mathrm{FP}} / \sqrt{\bar{g}}= & i \delta_{B}\left[\bar{C}_{\mu}\left(F^{\mu}+\frac{a}{2 Z_{\mathrm{GF}}} B^{\mu}\right)\right] / \delta \lambda \\
= & -B_{\mu}\left(F^{\mu}+\frac{a}{2 Z_{\mathrm{GF}}} B^{\mu}\right) \\
& +i \bar{C}_{\mu}\left[\overline { \nabla } _ { \alpha } \left(\bar{\nabla}^{\mu} C^{\alpha}+\bar{\nabla}^{\alpha} C^{\mu}\right.\right. \\
& \left.-\frac{2 m}{1+d m} \bar{g}^{\mu \alpha} \bar{\nabla}_{\rho} C^{\rho}\right) \\
& \left.-\frac{1+\bar{b}}{d} \bar{\nabla}^{\mu}\left(\frac{2}{1+d m} \bar{\nabla}_{\rho} C^{\rho}\right)\right] \\
= & -\frac{a}{2 Z_{\mathrm{GF}}} \tilde{B}_{\mu} \tilde{B}^{\mu}+\frac{Z_{\mathrm{GF}}}{2 a} F_{\mu} F^{\mu} \\
& +i \bar{C}_{\mu} \Delta^{(g h) \mu}{ }_{\nu} C^{\nu},
\end{aligned}
$$

and we have set $\bar{b}=(1+d m) b$ and defined

$$
\begin{aligned}
\tilde{B}_{\mu} & =B_{\mu}+\frac{Z_{\mathrm{GF}}}{a} F_{\mu}, \\
\Delta_{\mu \nu}^{(g h)} & \equiv \bar{g}_{\mu \nu} \square+\left(1-2 \frac{b+1}{d}\right) \bar{\nabla}_{\mu} \bar{\nabla}_{\nu}+\bar{R}_{\mu \nu} .
\end{aligned}
$$

Here $a$ is another dimensionless gauge parameter, and $Z_{\mathrm{GF}}$ is a parameter with dimension $d-2$. Since the $B_{\mu}$ field involves no derivatives, we can simply integrate it out and then we are left with the gauge-fixing and FP ghost terms.

Using the York decomposition,

$$
\begin{aligned}
F_{\mu}= & -\left(\Delta_{L 1}-\frac{2 \bar{R}}{d}\right) \xi_{\mu} \\
& -\bar{\nabla}_{\mu}\left(\frac{d-1}{d}\left(\Delta_{L 0}-\frac{\bar{R}}{d-1}\right) \sigma+\frac{\bar{b}}{d} h\right) .
\end{aligned}
$$

Inserting this into the gauge-fixing term in (2.52), integrating by parts, rewriting in terms of Lichnerowicz Laplacians and using the York decomposition, we obtain the gaugefixing term

$$
\begin{aligned}
S_{\mathrm{GF}}= & -\frac{Z_{\mathrm{GF}}}{2 a} \int d^{d} x \sqrt{\bar{g}}\left[\hat{\xi}_{\mu}\left(\Delta_{L 1}-\frac{2 \bar{R}}{d}\right) \hat{\xi}^{\mu}\right. \\
& +\frac{(d-1)^{2}}{d^{2}} \hat{\sigma}\left(\Delta_{L 0}-\frac{\bar{R}}{d-1}\right) \hat{\sigma} \\
& +\frac{(d-1) \bar{b}}{d^{2}} \hat{\sigma} \sqrt{\Delta_{L 0}} \sqrt{\left.\Delta_{L 0}-\frac{\bar{R}}{d-1} h+\frac{\bar{b}^{2}}{d^{2}} h \Delta_{L 0} h\right] .}
\end{aligned}
$$

We see that a specific combination of scalar degrees of freedom appears in the gauge-fixing term. It is sometimes 
convenient to write the scalar sector in terms of the gaugeinvariant variable $s$ defined in Eq. (2.40) and this new degree of freedom which, in terms of the original fields, is given by

$$
\begin{aligned}
\chi= & \sigma+\frac{b}{(d-1-b) \Delta_{L 0}-\bar{R}} s=\frac{(d-1) \Delta_{L 0}-\bar{R}}{(d-1-b) \Delta_{L 0}-\bar{R}} \sigma \\
& +\frac{b(1+d m)}{(d-1-b) \Delta_{L 0}-\bar{R}} h .
\end{aligned}
$$

A short calculation shows that the Jacobian of the transformation $(\sigma, h) \rightarrow(s, \chi)$ is 1 .

In terms of the new variable, the gauge-fixing action is

$$
\begin{aligned}
S_{\mathrm{GF}}= & -\frac{Z_{\mathrm{GF}}}{2 a} \int d^{d} x \sqrt{\bar{g}}\left[\xi_{\mu}\left(\Delta_{L 1}-\frac{2 \bar{R}}{d}\right)^{2} \xi^{\mu}\right. \\
& \left.+\frac{(d-1-b)^{2}}{d^{2}} \chi \Delta_{L 0}\left(\Delta_{L 0}-\frac{\bar{R}}{d-1-b}\right)^{2} \chi\right] .
\end{aligned}
$$

From (2.56) we see that $\chi$ transforms in the same way as $\sigma$. Thus $\xi$ and $\chi$ can be viewed as the gauge degrees of freedom and $h^{T T}$ and $s$ as the physical degrees of freedom. We will use this parametrization later.

The ghost action (2.52) for this gauge fixing contains a non-minimal operator [1]. We also decompose the ghost into transverse and longitudinal parts

$C_{v}=C_{v}^{T}+\bar{\nabla}_{\nu} C^{L}=C_{v}^{T}+\bar{\nabla}_{v} \frac{1}{\sqrt{-\bar{\nabla}^{2}}} C^{L}$,

and the same for $\bar{C}_{\mu}$. (This change of variables has unit Jacobian.) The ghost action then splits in two terms

$$
\begin{aligned}
S_{g h}= & i \int d^{d} x \sqrt{\bar{g}}\left[\bar{C}^{T \mu}\left(\Delta_{L 1}-\frac{2 \bar{R}}{d}\right) C_{\mu}^{T}\right. \\
& \left.+2 \frac{d-1-b}{d} \bar{C}^{\prime L}\left(\Delta_{L 0}-\frac{\bar{R}}{d-1-b}\right) C^{\prime L}\right] .
\end{aligned}
$$

Note for future reference that the gauge-fixing condition and the ghost action are pathological for $b=d-1$.

Let us observe that $Z_{\mathrm{GF}}$ appears in the combination $Z_{\mathrm{GF}} / a$, where $a$ is a dimensionless gauge parameter, while $Z_{\mathrm{GF}}$ is a constant of dimension $d-2$. There are then two natural options regarding the constant $Z_{\mathrm{GF}}$. The first choice is to treat it as a fixed parameter (later we shall identify it with a power of the cutoff). This leads to simpler formulas for many expressions, and we shall use it extensively later. It is, however, not appropriate for the discussion of perturbative Einstein gravity. The reason is that in the limit $G \rightarrow 0$ the coefficient $Z_{N}$ of the Hessian diverges. If we keep $Z_{\mathrm{GF}}$ and $a$ constant in the limit, then the gauge-fixing term becomes negligible relative to the rest of the quadratic action. The gauge fluctuations remain unsuppressed and one can anticipate divergences. This is exactly what happens, as we shall mention later on. One can compensate the behavior of $Z_{N}$ by keeping $Z_{\mathrm{GF}}$ fixed and letting simultaneously $a \rightarrow 0$. Alternatively, one can set $Z_{\mathrm{GF}}=Z_{N}$. In this case, in the Gaussian limit $G \rightarrow 0$, the kinetic terms of the gauge-invariant and gauge degrees of freedom scale in the same way and one obtains sensible results for all values of $a$ and $b$.

In the following we shall discuss also different choices for the gauge fixing. One is the so-called "unimodular physical gauge", where one sets $\hat{\xi}_{\mu}=0$ and $h=0$. As shown in [27], this is equivalent to the above standard gauge in the limit $b \rightarrow \pm \infty$ and $a \rightarrow 0$.

\subsection{Four-derivative gauge-fixing terms}

In HDG it is customary to use gauge-fixing terms that contain four derivatives. In order to further check the gauge independence of the results, in Sect. 3.2 we consider the gauge fixing in our previous paper [24] but now with arbitrary gauge parameters.

We can simply repeat the same procedure to derive the gauge-fixing and FP terms but with additional higherderivative operator

$Y_{\mu \nu} \equiv \bar{g}_{\mu \nu} \square+c \bar{\nabla}_{\mu} \bar{\nabla}_{\nu}-f \bar{\nabla}_{\nu} \bar{\nabla}_{\mu}$,

where $c$ and $f$ are additional gauge parameters:

$$
\begin{aligned}
\mathcal{L}_{\mathrm{GF}+\mathrm{FP}} / \sqrt{\bar{g}}= & i \delta_{B}\left[\bar{C}_{\mu} Y^{\mu \nu}\left(F_{v}-\frac{a}{2 Z_{\mathrm{GF}}} B_{v}\right)\right] / \delta \lambda \\
= & -\frac{a}{2 Z_{\mathrm{GF}}} \tilde{B}_{\mu} Y^{\mu \nu} \tilde{B}_{v}+\frac{Z_{\mathrm{GF}}}{2 a} F_{\mu} Y^{\mu \nu} F_{\nu} \\
& +i \bar{C}_{\mu} Y^{\mu \nu} \Delta_{v \rho}^{(g h)} C^{\rho},
\end{aligned}
$$

where the gauge-fixing function $F_{\mu}$ is defined in (2.47), and $\tilde{B}_{\mu}$ and $\Delta_{\mu \nu}^{(g h)}$ in (2.53). Here we should notice that the dimension of the constant $Z_{\mathrm{GF}}$ is changed to $d-4$.

Note also that the field $B_{\mu}$ was an auxiliary field in the two-derivative gauge-fixing case (2.52), but here it is dynamical. With higher-derivative gauge fixing, quite often only the contribution $\Delta_{\mu \nu}^{(g h)}$ is incorporated but that from $Y^{\mu \nu}$ in the FP ghost kinetic term is ignored, and then it is claimed that somehow the contribution from the "third ghost" $-\frac{1}{2} \log \left(\operatorname{det}\left(Y_{\mu \nu}\right)\right)$ must be added. We see here that this is automatic in the BRST invariant formulation, because we have contributions $-\log \left(\operatorname{det}\left(Y^{\mu \nu}\right)\right)$ from the FP ghost kinetic term and $\frac{1}{2} \log \left(\operatorname{det}\left(Y^{\mu \nu}\right)\right)$ from the field $B_{\mu}$, giving the same result.

Using the York decomposition (2.24), we can calculate the contributions without fixing gauge parameters and so can check the gauge dependence directly here. We find that the 
terms (2.61) are cast into

$$
\begin{aligned}
& -\frac{a}{2 Z_{\mathrm{GF}}}\left[B_{\mu}^{T}\left(\Delta_{L 1}-\frac{1-f}{d} \bar{R}\right) B^{\mu T}\right. \\
& \left.+B^{L} \Delta_{L 0}\left\{(1+c-f) \Delta_{L 0}-\frac{1-f}{d} \bar{R}\right\} B^{L}\right] \\
& +\frac{Z_{\mathrm{GF}}}{2 a}\left[\xi_{\mu}\left(\Delta_{L 1}-\frac{2}{d} \bar{R}\right)^{2}\left(\Delta_{L 1}-\frac{1-f}{d} \bar{R}\right) \xi^{\mu}\right. \\
& +\left(\frac{d-1-b}{d}\right)^{2} \chi \Delta_{L 0}\left(\Delta_{L 0}-\frac{\bar{R}}{d-1-b}\right)^{2} \\
& \left.\left.\times\left\{(1+c-f) \Delta_{L 0}-\frac{1-f}{d} \bar{R}\right\} \chi\right] \bar{R}\right)\left(\Delta_{L 1}-\frac{2}{d} \bar{R}\right) C^{\mu T} \\
& +i \bar{C}_{\mu}^{T}\left(\Delta_{L 1}-\frac{1-f}{d} \bar{R}\right) \\
& +2 i \frac{d-1-b}{d} \bar{C}^{L} \Delta_{L 0}\left(\Delta_{L 0}-\frac{\bar{R}}{d-1-b}\right) \\
& \quad \times\left\{(1+c-f) \Delta_{L 0}-\frac{1-f}{d} \bar{R}\right\} C^{L},
\end{aligned}
$$

where we have defined transverse and longitudinal parts of the $\tilde{B}_{\mu}$ and FP ghosts $\bar{C}_{\mu}$ and $C_{\mu}$, as usual.

In Sect. 3.1 we will discuss the gauge independence of the theory in the general quadratic gauge. In Sect. 4.5 we will restrict ourselves to the class of gauges where $c=f=1$ but with generic $a, b$. This is equivalent to inserting a Lichnerowicz Laplacian $\Delta_{L 1}$ in the quadratic gauge-fixing term (2.52), or in other words to set $Y^{\mu \nu}=\bar{g}^{\mu \nu} \Delta_{L 1}$. After performing the York decomposition, this yields additional factors $\Delta_{L 1}$ and $\Delta_{L 0}$ in the quadratic actions of $\xi_{\mu}$ and $\chi$, Eq. (2.57). The resulting additional determinants are offset by the determinant of the operator coming from the $B_{\mu}$ sector, as will become clear in the following.

\section{Universality on-shell in $d=4$}

In this section we consider the theory in $d=4$ on an Einstein background. As noted in Sect. 2.1, if we put $Z_{N}=0$, the equation of motion of HDG is automatically satisfied for $d=4$, so one would expect the effective action to be gaugeand parametrization-independent. We will check that this is indeed the case at the formal level of determinants. In the following section we will have a more explicit check of this property in the expressions for the divergences.

\subsection{General two-derivative gauge fixing}

The Hessian of pure higher-derivative gravity in $d=4$ has the simple form (2.45), independent of the choice of parametrization. It is expressed entirely in terms of the gaugeinvariant variables $h^{T T}$ and $s$. These fields do not appear in the gauge-fixing action, so their contribution to the one-loop effective action is given by

$$
\begin{aligned}
& \operatorname{Det}\left(\Delta_{L 2}-\frac{\bar{R}}{2}\right)^{-1 / 2} \operatorname{Det}\left(\Delta_{L 2}-\left(1+\frac{2 \alpha}{\beta}\right) \bar{R}\right)^{-1 / 2} \\
& \operatorname{Det} \Delta_{L 0}^{-1 / 2} \operatorname{Det}\left(\Delta_{L 0}-\frac{\bar{R}}{3}\right)^{-1 / 2} .
\end{aligned}
$$

We choose the gauge as in Sect. 2.6. It is convenient to express the gauge-fixing term as in (2.57). The fields $\xi$ and $\chi$ contribute to the one-loop action the terms (as ordered):

$\operatorname{Det}\left(\Delta_{L 1}-\frac{\bar{R}}{2}\right)^{-1} \operatorname{Det} \Delta_{L 0}^{-1 / 2} \operatorname{Det}\left(\Delta_{L 0}-\frac{\bar{R}}{3-b}\right)^{-1}$

The ghost action (2.59) gives the determinants

$\operatorname{Det}\left(\Delta_{L 1}-\frac{\bar{R}}{2}\right) \operatorname{Det}\left(\Delta_{L 0}-\frac{\bar{R}}{3-b}\right)$,

and finally the Jacobians of the change of variables from $h_{\mu \nu}$ to $h_{\mu \nu}^{T T}, \xi^{\mu}, \sigma$ and $h$ is

$\operatorname{Det}\left(\Delta_{L 1}-\frac{\bar{R}}{2}\right)^{1 / 2} \operatorname{Det} \Delta_{L 0}^{1 / 2} \operatorname{Det}\left(\Delta_{L 0}-\frac{\bar{R}}{3}\right)^{1 / 2}$.

Note that the gauge parameter $a$ only appears in the Hessian in the prefactors and not in the operators. The only dependence on the gauge parameter $b$ is in two determinants that cancel. This proves the gauge independence of the one-loop action. The final result is

$\frac{\sqrt{\operatorname{Det}\left(\Delta_{L 1}-\frac{\bar{R}}{2}\right)}}{\sqrt{\operatorname{Det}\left(\Delta_{L 2}-\frac{\bar{R}}{2}\right)} \sqrt{\operatorname{Det}\left(\Delta_{L 2}-\left(1+\frac{2 \alpha}{\beta}\right) \bar{R}\right)} \sqrt{\operatorname{Det} \Delta_{L 0}}}$.

\subsection{General four-derivative gauge fixing}

If we use the general four-derivative gauge fixing of Sect. 2.7, we have again the determinants (3.1) coming from the physical degrees of freedom and (3.4) coming from the Jacobians. The determinants coming from the fields $\xi_{\mu}$ and $\chi$ are

$$
\begin{aligned}
& \operatorname{Det}\left(\Delta_{L 1}-\frac{\bar{R}}{2}\right)^{-1} \operatorname{Det}\left(\Delta_{L 1}-\frac{1-f}{4} \bar{R}\right)^{-1 / 2} \\
& \quad \times \operatorname{Det}\left(\Delta_{L 0}-\frac{\bar{R}}{3-b}\right)^{-1} \\
& \quad \times \operatorname{Det}\left(\Delta_{L 0}-\frac{1-f}{4(1+c-f)} \bar{R}\right)^{-1 / 2}
\end{aligned}
$$


the ghosts give

$$
\begin{gathered}
\operatorname{Det}\left(\Delta_{L 1}-\frac{\bar{R}}{2}\right) \operatorname{Det}\left(\Delta_{L 1}-\frac{1-f}{4} \bar{R}\right) \\
\times \operatorname{Det} \Delta_{L 0} \operatorname{Det}\left(\Delta_{L 0}-\frac{\bar{R}}{3-b}\right) \\
\times \operatorname{Det}\left(\Delta_{L 0}-\frac{1-f}{4(1+c-f)} \bar{R}\right),
\end{gathered}
$$

and finally we have the contributions of the $B_{\mu}$ field

$$
\begin{aligned}
& \operatorname{Det}\left(\Delta_{L 1}-\frac{1-f}{4} \bar{R}\right)^{-1 / 2} \operatorname{Det} \Delta_{L 0}^{-1 / 2} \\
& \times \operatorname{Det}\left(\Delta_{L 0}-\frac{1-f}{4(1+c-f)} \bar{R}\right)^{-1 / 2} .
\end{aligned}
$$

Putting everything together, we see that all the terms depending on the gauge parameters cancel out and the remaining ones give again (3.5). Thus we have explicitly shown, at this formal level, that the results are completely gauge independent.

\subsection{Physical gauge}

In the calculation of Sect. 3.1, and even more in Sect. 3.2, there is a large number of cancellations between various determinants. Consider instead the "physical" gauge $\hat{\xi}_{\mu}=0$, $h=0$, discussed in [27,28]. It leaves only the fields $h^{T T}$ and $\hat{\sigma}$, and no Jacobians. The Hessians of $h^{T T}$ and $\hat{\sigma}$ are given by (2.35) and (2.37), respectively. There are a real scalar ghost and a real transverse vector ghost, with ghost operators $\Delta_{L 0}$ and $\Delta_{L 1}-R / 2$. Putting together these terms, one immediately obtains the effective action (3.5). In fact this is the most direct way of getting it, because there is no cancellation of determinants between unphysical degrees of freedom and ghosts.

\subsection{The conformal case}

Now we consider the conformal case where $\beta / \alpha=-3$. The effective action in this case cannot be simply obtained as a particular case of Eq. (3.5), because the action is invariant also under Weyl transformations and this requires a separate gauge fixing.

The Hessian is only nonzero in the spin-2 sector and is given by (2.46). For the Weyl invariance we can gauge fix $h=0$, without any ghost because $h$ transforms under Weyl transformations by a shift. For diffeomorphisms we choose a standard gauge fixing of the form (2.55). Since $h=0$, the value of $b$ is immaterial. Equation (2.56) is replaced by $\chi=\sigma$, so the decomposition of the gauge-fixing and ghost actions, and the corresponding determinants, are the same as in Sect. 2.6, with $b=0$.
We use the fields $\hat{\xi}$ and $\hat{\sigma}$ (see (2.25)) in such a way that no Jacobian is needed. The one-loop effective action is

$$
\frac{\sqrt{\operatorname{Det}\left(\Delta_{L 1}-\frac{\bar{R}}{2}\right)} \sqrt{\operatorname{Det}\left(\Delta_{L 0}-\frac{\bar{R}}{3}\right)}}{\sqrt{\operatorname{Det}\left(\Delta_{L 2}-\frac{\bar{R}}{2}\right)} \sqrt{\operatorname{Det}\left(\Delta_{L 2}-\frac{\bar{R}}{3}\right)}}
$$

The two terms in the denominator come from the TT part. The terms in the numerator come from the ghosts (power 1 ) and from the gauge-fixing term (power $-1 / 2$ ). Note that there is no dependence on the gauge parameter $a$. It only appears in the prefactors of the quadratic action, which drop out.

Alternatively we can choose a different gauge. For Weyl transformations we still choose $h=0$, which leaves no ghost term. For diffeomorphisms we choose the second type of physical gauge explained in the end of section III.B of [27], namely $\hat{\sigma}=0$ and $\hat{\xi}=0$. This is equivalent to taking the Landau gauge limit $a \rightarrow 0$. The ghosts are a real scalar and a real transverse vector and the ghost operators are $\Delta_{L 0}-\bar{R} / 3$ and $\Delta_{L 1}-\bar{R} / 2$. The effective action is given again by (3.10).

\subsection{The Einstein-Hilbert case}

Finally we consider the Einstein-Hilbert theory, where we put $\alpha=\beta=0$. In this case it is natural to use the twoderivative gauge fixing. In order to maintain the expressions to a manageable size we write them here for the case $\omega=$ $m=0$. Then from the decompositions in Sects. 2.5 and 2.6 we find that the gauge-invariant variable $h^{T T}$ gives

$\operatorname{Det}\left(\Delta_{L 2}-\frac{\bar{R}}{2}+\frac{1}{2} \bar{E}\right)^{-1 / 2}$;

the spin-1 field $\xi_{\mu}$ gives

$\operatorname{Det}\left(\Delta_{L 1}+\frac{a-1}{2} \bar{R}-2 a \Lambda\right)^{-1 / 2}$

the scalars $\sigma$ and $h$ give

$$
\begin{aligned}
& \operatorname{Det}\left(\Delta_{L 0}^{2}+\frac{\left(4 \Lambda\left(-2 a+b^{2}-3\right)+R\left(2 a-b^{2}+2 b-3\right)\right)}{(b-3)^{2}} \Delta_{L 0}\right. \\
& \left.+\frac{4 \Lambda(4 a \Lambda-a R+R)}{(b-3)^{2}}\right),
\end{aligned}
$$

and finally the ghosts give

$\operatorname{Det}\left(\Delta_{L 1}-\frac{\bar{R}}{2}\right) \operatorname{Det}\left(\Delta_{L 0}-\frac{\bar{R}}{3-b}\right)$.

Putting all together, and making the replacements $a \rightarrow 1 / \gamma$ and $b \rightarrow \beta$, we find that it agrees with Eq. (4.4) of [29]. If 
we go on shell by putting $\bar{R}=4 \Lambda$ the scalar contribution cancels the scalar ghost determinant, leaving just

$$
\frac{\sqrt{\operatorname{Det}\left(\Delta_{L 1}-\frac{\bar{R}}{2}\right)}}{\sqrt{\operatorname{Det}\left(\Delta_{L 2}-\frac{\bar{R}}{2}\right)}},
$$

which is gauge independent and agrees with the classic result of [30]. A slightly more complicated calculation shows that also the dependence on the parameters $\omega$ and $m$ automatically goes away on shell.

\section{The divergences}

\subsection{Derivation}

The one-loop effective action contains a divergent part

$$
\begin{aligned}
\Gamma_{k}= & \int d^{d} x \sqrt{\bar{g}}\left[\frac{A_{1}}{16 \pi d} k^{d}+\frac{B_{1}}{16 \pi(d-2)} k^{d-2} \bar{R}\right. \\
& +\beta_{\alpha} \frac{k^{d-4}}{d-4} \bar{R}^{2}+\beta_{\beta} \frac{k^{d-4}}{d-4} \bar{R}_{\mu \nu} \bar{R}^{\mu \nu} \\
& \left.+\beta_{\gamma} \frac{k^{d-4}}{d-4} \bar{R}_{\mu \nu \rho \sigma} \bar{R}^{\mu \nu \rho \sigma}\right],
\end{aligned}
$$

where $k$ stands for a cutoff. In $d=4$, the power divergences $k^{d-4} /(d-4)$ of the last three terms are replaced by terms $\log (k / \mu)$, where we introduced a reference mass scale $\mu$. In general one would have separate Riemann squared, Ricci squared and $R^{2}$ terms, but on an Einstein space (2.8) the latter two merge into a single term proportional to $\bar{R}^{2}$. Then we will denote

$C_{1}=\beta_{\alpha}+\frac{1}{d} \beta_{\beta}$,

the coefficient of the $\bar{R}^{2}$ divergence and $D_{1}=\beta_{\gamma}$ the coefficient of the $\bar{R}_{\mu \nu \rho \sigma} \bar{R}^{\mu \nu \rho \sigma}$ divergence. We note that in I we used a maximally symmetric background, where $\bar{R}_{\mu \nu \rho \sigma} \bar{R}^{\mu \nu \rho \sigma}=\frac{2}{d(d-1)} \bar{R}^{2}$ Thus, the coefficient $C_{1}$ of I corresponds to the combination $C_{1}+\frac{2}{d(d-1)} D_{1}$.

We describe here the algorithm that we use to derive the coefficients. Instead of $\Gamma_{k}$ we shall evaluate the derivative [31,32]:

$$
\begin{aligned}
\dot{\Gamma}_{k}= & \int d^{d} x \sqrt{\bar{g}}\left[\frac{A_{1}}{16 \pi} k^{d}+\frac{B_{1}}{16 \pi} k^{d-2} \bar{R}\right. \\
& \left.+\left(\beta_{\alpha} \bar{R}^{2}+\beta_{\beta} \bar{R}_{\mu \nu} \bar{R}^{\mu \nu}+\beta_{\gamma} \bar{R}_{\mu \nu \rho \sigma} \bar{R}^{\mu \nu \rho \sigma}\right) k^{d-4}+\ldots\right],
\end{aligned}
$$

where the overdot stands for $k \frac{d}{d k}$. The advantage of this procedure is that this quantity is convergent. Independently of the renormalizability properties of the theory, it defines a flow on the space of all couplings that are permitted by the symmetries of the system. The divergences of (4.1) in the limit $k \rightarrow \infty$ are then found by integrating the differential equation (4.3) from some initial scale $k_{0}$ up to $k$. The coefficients $A_{1}, B_{1}, \beta_{\alpha}, \beta_{\beta}$ and $\beta_{\gamma}$ enter in the beta functions of the couplings $\Lambda, G, \alpha, \beta, \gamma$, but we postpone a discussion of this point to Sect. 7 .

The technique employed to evaluate the right hand side of (4.3) is similar to the one described in I, but this time the cutoff is taken to be a function of the Lichnerowicz Laplacians instead of the Bochner Laplacian $-\bar{\nabla}^{2}$, as in I. In each spin sector, the Lichnerowicz operator $\Delta_{L}$ is replaced by the regularized one $P_{k}\left(\Delta_{L}\right)=\Delta_{L}+R_{k}\left(\Delta_{L}\right)$, where we use the optimized cutoff $R_{k}\left(\Delta_{L}\right)=\left(k^{2}-\Delta_{L}\right) \theta\left(k^{2}-\Delta_{L}\right)$. Then $\dot{\Gamma}_{k}$ is given by

$$
\begin{aligned}
\dot{\Gamma}_{k}= & \frac{1}{2} \operatorname{Tr}\left(\frac{\dot{\Delta}_{k}^{(2)}}{\Delta_{k}^{(2)}}\right)+\frac{1}{2} \operatorname{Tr}\left(\frac{\dot{\Delta}_{k}^{(1)}}{\Delta_{k}^{(1)}}\right)+\frac{1}{2} \operatorname{Tr}\left(\frac{\dot{\Delta}_{k}^{(0)}}{\Delta_{k}^{(0)}}\right) \\
& -\operatorname{Tr}\left(\frac{\dot{\Delta}_{g h, k}^{(1)}}{\Delta_{g h, k}^{(1)}}\right)-\operatorname{Tr}\left(\frac{\dot{\Delta}_{g h, k}^{(0)}}{\Delta_{g h, k}^{(0)}}\right),
\end{aligned}
$$

where $\Delta_{k}$ are the kinetic operators that appear in each spin sector and the numerator is $\dot{\Delta}_{k}=\dot{R}_{k}\left(\Delta_{L}\right)=2 k^{2} \theta\left(k^{2}-\Delta_{L}\right)$. The step function cuts off the trace to eigenvalues of $\Delta_{L}$ that are smaller than $k^{2}$. For example in the spin-2 sector

$$
\begin{aligned}
\frac{1}{2} \operatorname{Tr} & \left(\frac{\dot{\Delta}_{k}^{(2)}}{\Delta_{k}^{(2)}}\right)=\frac{1}{2} \frac{1}{(4 \pi)^{d / 2}} \\
\times & {\left[W ( \Delta _ { L } ^ { ( 2 ) } , 0 ) \left(Q_{d / 2} b_{0}\left(\Delta_{L}^{(2)}\right)+Q_{d / 2-1} b_{2}\left(\Delta_{L}^{(2)}\right)\right.\right.} \\
& \left.+Q_{d / 2-2} b_{4}\left(\Delta_{L}^{(2)}\right)\right)+W^{\prime}\left(\Delta_{L}^{(2)}, 0\right) \bar{R}\left(Q_{d / 2} b_{0}\left(\Delta_{L}^{(2)}\right)\right. \\
& \left.+Q_{d / 2-1} b_{2}\left(\Delta_{L}^{(2)}\right)\right)+\frac{1}{2} W^{\prime \prime} \\
& \left.\times\left(\Delta_{L}^{(2)}, 0\right) \bar{R}^{2}\left(Q_{d / 2} b_{0}\left(\Delta_{L}^{(2)}\right)\right)+\ldots\right]
\end{aligned}
$$

where $W\left(\Delta_{L}^{(2)}, \bar{R}\right)=\frac{\dot{\Delta}_{L k}^{(2)}}{\Delta_{L k}^{(2)}}$ and primes denote derivatives with respect to $\bar{R}$. The coefficients $Q_{n}$ and the heat kernel coefficients $b_{n}$ are listed in Appendix A.

Similar formulas hold for the spin- 1 and spin- 0 sectors and for the ghosts. In the scalar term, $\Delta_{k}^{(0)}$ is a two-by-two matrix, and the fraction has to be understood as the product of $\dot{\Delta}_{k}^{(0)}$ with the inverse of $\Delta_{k}^{(0)}$. The functional trace thus involves also a trace over this two-by-two matrix. With these data one can write the expansion of (4.4) in powers of $\bar{R}$, and comparing with (4.3) one can read off the coefficients $A_{1}, B_{1}$ and $C_{1}$.

\subsection{Results}

From now on we will deal with dimensionless variables $\tilde{\alpha}=k^{4-d} \alpha, \quad \tilde{\beta}=k^{4-d} \beta, \quad \tilde{\Lambda}=k^{-2} \Lambda$, 
$\tilde{G}=k^{d-2} G, \quad\left(\tilde{Z}_{N}=k^{2-d} Z_{N}\right)$,

and

$\tilde{Z_{\mathrm{GF}}}=\left\{\begin{array}{l}k^{2-d} Z_{\mathrm{GF}} \text { for two-derivative gauge fixing (2.52), } \\ k^{4-d} Z_{\mathrm{GF}} \text { for four-derivative gauge fixing (2.61). }\end{array}\right.$

In I, the couplings $\tilde{\alpha}$ and $\tilde{\beta}$ were absent, and $\tilde{G}$ only appeared in the Hessian through the overall prefactor $Z_{N}$. Since this prefactor canceled between numerator and denominator in (4.4), the divergences were independent of $\tilde{G}$. This does no longer happen in the present theory, so the coefficients $A_{1}, B_{1}$ and $C_{1}$ depend in general on the dimension $d$, on the measure parameters $m$ and $\omega$, on the gauge parameters $a$ and $b$, and on the couplings $\tilde{\Lambda}, \tilde{G}, \tilde{\alpha}$ and $\tilde{\beta}$. The general expressions for the coefficients of the divergences are extremely complicated and not instructive. We will therefore only write them in certain limits where they simplify enough.

Compared to I, we have to take into account the additional dependence on the couplings $\tilde{G}, \tilde{\alpha}$ and $\tilde{\beta}$. We will consider two limiting situations. One is the limit $\tilde{\alpha} \rightarrow 0$ and $\tilde{\beta} \rightarrow 0$. In this case the beta functions should reduce to those of Einstein-Hilbert gravity and match with those of I (modulo scheme dependences, due to the different form of the cutoff in this paper). Still, the present results are more general because we consider a generic Einstein background, which allows us to distinguish at least two of the higher-derivative couplings, whereas in I a maximally symmetric background was used, allowing us to calculate the coefficient of a single combination of the higher-derivative couplings. We will refer to this as the "Einstein-Hilbert limit" (EH limit). ${ }^{2}$

The other limit consists in taking $\tilde{Z}_{N} \rightarrow 0$, which is equivalent to $\tilde{G} \rightarrow \infty$. In this case one is left with functions of the higher-derivative couplings only. We will refer to this as the "four-derivative gravity limit" (4DG-limit).

There is still the freedom of choosing between the twoderivative and four-derivative gauges. In Sects. 4.3 and 4.4 we will consider the 4DG- and the EH-limits of the theory, using the two-parameter family of two-derivative gauges introduced in Sect. 2.6. In Sect. 4.5 we will discuss the changes that occur when using the two-parameter family of four-derivative gauges introduced in Sect. 2.7. It will turn out that in order to take the EH- and 4DG-limits, different choices have to be made regarding the overall gauge-fixing coefficient $Z_{\mathrm{GF}}$. These are spelled out in detail in the following sections.

2 The same results can be obtained, at least in some cases, by taking the limit $\tilde{Z}_{N} \rightarrow \infty$, which is the same as considering the perturbative regime $\tilde{G} \rightarrow 0$.

\subsection{The 4DG-limit $\left(\tilde{Z}_{N} \rightarrow 0\right)$}

For this calculation we set $\tilde{Z_{\mathrm{GF}}}=1$. The coefficients $A_{1}, B_{1}$ and $D_{1}$ turn out to be universal (i.e. independent of the gauge and parametrization) in any dimension:

$$
\begin{aligned}
A_{1}= & \frac{8(d-2)}{(4 \pi)^{d / 2-1} \Gamma(d / 2)}, \\
B_{1}= & \frac{2}{(4 \pi)^{d / 2-1} 3 d^{2} \tilde{\beta}(4(d-1) \tilde{\alpha}+d \tilde{\beta}) \Gamma(d / 2)} \\
& \times\left[48 d\left(d^{3}-2 d^{2}-d+2\right) \tilde{\alpha}^{2}\right. \\
& +4\left(d^{5}-12 d^{4}+41 d^{3}-102 d^{2}+36 d+48\right) \tilde{\alpha} \tilde{\beta} \\
& \left.+\left(d^{5}-14 d^{4}+30 d^{3}-60 d^{2}-72 d+96\right) \tilde{\beta}^{2}\right], \\
D_{1}= & \frac{-1050+589 d-34 d^{2}+d^{3}}{(4 \pi)^{d / 2} 360 \Gamma(d / 2)} .
\end{aligned}
$$

The coefficient $C_{1}$ has a complicated dependence on $m, \omega$, $a, b, \tilde{\alpha}$ and $\tilde{\beta}$, which we do not report here. A special case will be given below in (4.20). However, in $d=4$ all four coefficients, including $C_{1}$, are universal:

$$
\begin{aligned}
A_{1} & =\frac{4}{\pi}, \\
B_{1} & =\frac{15 \tilde{\alpha}-14 \tilde{\beta}}{3 \pi \tilde{\beta}}, \\
C_{1} & =\frac{1200 \tilde{\alpha}^{2}+200 \tilde{\alpha} \tilde{\beta}-183 \tilde{\beta}^{2}}{1920 \pi^{2} \tilde{\beta}^{2}}, \\
D_{1} & =\frac{413}{2880 \pi^{2}} .
\end{aligned}
$$

This is an explicit consequence of the statement, made in Sect. 3.1, that the one-loop effective action of higherderivative gravity is independent of the parametrization and gauge choice in $d=4$.

The expressions for $C_{1}$ and $D_{1}$ given above are in agreement with the standard results for the beta functions in HDG $[9,10,33]$ (also derived by means of (4.4) in [24,34-36]):

$$
\begin{aligned}
& \beta_{\tilde{\alpha}}=\frac{1}{(4 \pi)^{2}} \frac{90 \tilde{\alpha}^{2}-23 \tilde{\beta}^{2}-338 \tilde{\gamma}^{2}+15 \tilde{\alpha} \tilde{\beta}-120 \tilde{\alpha} \tilde{\gamma}-199 \tilde{\beta} \tilde{\gamma}}{9(\tilde{\beta}+4 \tilde{\gamma})^{2}}, \\
& \beta_{\tilde{\beta}}=\frac{1}{(4 \pi)^{2}} \frac{371}{90}, \\
& \beta_{\tilde{\gamma}}=\frac{1}{(4 \pi)^{2}} \frac{413}{180} .
\end{aligned}
$$

Let us consider what happens if we choose $\tilde{Z_{\mathrm{GF}}}=\tilde{Z}_{N}$, as is usually done in Einstein-Hilbert theory. In the 4DGlimit, the coefficients $A_{1}$ and $D_{1}$ are still given by the formulas given above, but $B_{1}$ and $C_{1}$ are different, and gaugedependent. However, for the exponential parametrization $\left(\omega=\frac{1}{2}\right)$ in $d=4$, one gets the $m$-independent result 


$$
\begin{aligned}
& B_{1}=\frac{15 \tilde{\alpha}(b-3)^{2}+\tilde{\beta}\left(3 a-14(b-3)^{2}\right)}{3 \pi(b-3)^{2} \tilde{\beta}} \\
& C_{1}=\frac{\tilde{\beta}^{2}\left(240 a^{2}+40 a\left(b^{2}-18 b+45\right)-183(b-3)^{4}\right)+1200 \tilde{\alpha}^{2}(b-3)^{4}+200 \tilde{\alpha} \tilde{\beta}(b-3)^{4}}{1920 \pi^{2}(b-3)^{4} \tilde{\beta}^{2}} .
\end{aligned}
$$

We note that in the limit $a \rightarrow 0$ these reproduce the universal formulas given above. This observation agrees with the discussion in the end of Sect. 2.6. In the 4DG-limit, at fixed $k$, one sets $\tilde{Z}_{N}=0$. For fixed $a$ and $k$, and with the choice $\tilde{Z_{\mathrm{GF}}}=\tilde{Z}_{N}$, this implies that the gauge-fixing term vanishes too. The situation can be fixed by letting $a \rightarrow 0$, with the ratio $\tilde{Z_{\mathrm{GF}}} / a$ fixed and finite. In conclusion, the gauge dependence that occurs in (4.11) for $a \neq 0$ is an artifact of a bad gauge-fixing procedure. The correct result is given by (4.9).

This problem does not arise in the standard calculation of the beta functions of HDG in $d=4$, because there the gauge fixing is of the type considered in Sect. 3.2. When the gaugefixing term contains four derivatives, its overall coefficient is dimensionless in $d=4$ and there is never the temptation to introduce a factor $\tilde{Z}_{N}$.

\subsection{The EH limit}

Let us now consider the $\mathrm{EH}$ limit $\tilde{\alpha}, \tilde{\beta} \rightarrow 0$. In this case it is not appropriate to set $\tilde{Z_{\mathrm{GF}}}=1$, as we did in the preceding subsection, because of the issue in the gauge fixing discussed in the end of Sect. 2.6. Indeed if we insisted on this choice we would find that for $\tilde{\Lambda}=0$ only $A_{1}$ and $D_{1}$ are universal:

$$
\begin{aligned}
A_{1} & =\frac{4(d-3)}{(4 \pi)^{d / 2-1} \Gamma(d / 2)}, \\
D_{1} & =\frac{d^{3}-35 d^{2}+606 d-1080}{(4 \pi)^{d / 2} 720 \Gamma(d / 2)},
\end{aligned}
$$

whereas $B_{1}$ and $C_{1}$ would contain $\tilde{G}$ and diverge for $\tilde{G} \rightarrow 0$. For this reason we switch now to the choice $\tilde{Z_{\mathrm{GF}}}=\tilde{Z}_{N}$, which is more appropriate to discuss this limit.

We find that $A_{1}$ and $D_{1}$ are still given by (4.12). In $d=4$ we have

$$
\begin{aligned}
A_{1}= & \frac{1}{\pi} \\
B_{1}= & \frac{1}{12 \pi(b-3)^{2}(4 m+1)} \\
& \times\left[3 a \left(-18 b(4 m+1)^{2}(2 \omega-1)\right.\right. \\
& +3(2 \omega-1)(4 b m+b)^{2} \\
& +8 m(66(2 m+1) \omega-66 m-31)+70 \omega-31) \\
& +b^{2}(4 m+1)(72 m \omega-36 m+18 \omega-47) \\
& -6 b(4 m+1)(30(4 m+1) \omega-60 m-53) \\
& +9(48 m(10 m \omega-5 m+5 \omega-6)+26 \omega-55)],
\end{aligned}
$$

$D_{1}=\frac{53}{720 \pi^{2}}$.

We note that $\tilde{G}$ does not appear in these expressions, so that they have a smooth limit $\tilde{G} \rightarrow 0$. The expression for $C_{1}$ is still too long to be written but is likewise independent of $\tilde{G}$. We give it only for two cases: in the linear parametrization $m=\omega=0$ we have

$$
\begin{aligned}
C_{1}= & \frac{1}{1920 \pi^{2}(b-3)^{4}} \\
& \times\left[15 a^{2}\left(3 b^{4}-36 b^{3}+162 b^{2}-324 b+259\right)\right. \\
& -20 a\left(3 b^{4}-36 b^{3}+176 b^{2}-360 b+297\right) \\
& \left.+24\left(7 b^{4}-59 b^{3}+223 b^{2}-381 b+252\right)\right] .
\end{aligned}
$$

and in the exponential parametrization $\omega=1 / 2$, in which case the result is automatically independent of $m$, we have

$C_{1}=\frac{240 a^{2}+40 a\left(b^{2}-18 b+9\right)-3\left(29 b^{4}-348 b^{3}+1526 b^{2}-3372 b+2709\right)}{1920 \pi^{2}(b-3)^{4}}$.

One can plot these functions for fixed parametrization or for fixed gauge. One obtains plots that are very similar to those shown in I. They will not be repeated here.

We report for completeness the expressions $B_{1}$ and $C_{1}$ for $d=4$ in the "unimodular physical" gauge $b \rightarrow \pm \infty$, $a \rightarrow 0$, for $\tilde{\alpha}, \tilde{\beta}, \tilde{G}$ all tending to zero:

$B_{1}=\frac{36 m(2 \omega-1)+18 \omega-47}{12 \pi}$,

$C_{1}=\frac{180 m^{2}(1-2 \omega)^{2}+10 m\left(36 \omega^{2}-44 \omega+13\right)+45 \omega^{2}-65 \omega+14}{160 \pi^{2}}$.

\subsection{Results with the four-derivative gauge fixing}

In the preceding sections we used the two-derivative gaugefixing terms introduced in Sect. 2.6. We now discuss briefly the results when the four-derivative gauge-fixing terms of Sect. 2.7 are used instead.

The formal discussion of Sect. 3 indicates that in the 4DGlimit in four dimensions, the divergences should be universal. Explicit calculation confirms that in this case the coefficients $A_{1}, B_{1}, C_{1}, D_{1}$ are indeed given again by (4.9). Furthermore, the coefficients $A_{1}, B_{1}$ and $D_{1}$ are universal in any dimension and agree with those given in (4.8).

In the EH limit the coefficients $A_{1}$, and $D_{1}$ in $d=4$ are again as in (4.13), but the others are slightly different. For $B_{1}$ 
one has in general

$$
\begin{aligned}
B_{1}= & \frac{1}{12 \pi(b-3)^{2}(4 m+1)} \\
& \times\left[6 a \left(-18 b(4 m+1)^{2}(2 \omega-1)\right.\right. \\
& +3(2 \omega-1)(4 b m+b)^{2} \\
& +8 m(66(2 m+1) \omega-66 m-31)+70 \omega-31) \\
& +b^{2}(4 m+1)(72 m \omega-36 m+18 \omega-47) \\
& -6 b(4 m+1)(30(4 m+1) \omega-60 m-53) \\
& +9(48 m(10 m \omega-5 m+5 \omega-6)+26 \omega-55)] .
\end{aligned}
$$

Similarly the coefficient $C_{1}$ in the linear parametrization $m=$ $\omega=0$ is

$$
\begin{aligned}
C_{1}= & \frac{1}{1920 \pi^{2}(b-3)^{4}} \\
& \times\left[30 a^{2} t\left(3 b^{4}-36 b^{3}+162 b^{2}-324 b+259\right)\right. \\
& -5 a\left(15 b^{4}-180 b^{3}+898 b^{2}-1860 b+1575\right) \\
& \left.+24\left(7 b^{4}-59 b^{3}+223 b^{2}-381 b+252\right)\right],
\end{aligned}
$$

and in the exponential parametrization $\omega=1 / 2$ :

$C_{1}=\frac{480 a^{2}+80 a\left(b^{2}-15 b+9\right)-3\left(29 b^{4}-348 b^{3}+1526 b^{2}-3372 b+2709\right)}{1920 \pi^{2}(b-3)^{4}}$.

The result in the unimodular physical gauge $b \rightarrow \pm \infty, a \rightarrow$ 0 agrees with (4.16).

\subsection{Exponential parametrization and unimodular gauge}

It was found in I that, choosing the exponential parametrization $\omega=1 / 2$ and the unimodular gauge $b \rightarrow \pm \infty$, all dependence on the other two parameters $m$ and $a$, as well as the dependence on the cosmological constant $\tilde{\Lambda}$, drops out.

This result holds true also in the present context, in any dimension and independently of whether the gauge fixing contains two or four derivatives and independently of the treatment of the constant $Z_{\mathrm{GF}}$. The resulting coefficients are still too cumbersome to write, so we give them again only in two limits.

In the $4 \mathrm{DG}$-limit the coefficients $A_{1}, B_{1}$ and $D_{1}$ are given by (4.8) and

$$
\begin{aligned}
C_{1}= & \frac{1}{720(4 \pi)^{d / 2} d^{3} \tilde{\beta}^{2}(4(d-1) \tilde{\alpha}+d \tilde{\beta})^{2} \Gamma(d / 2)} \\
& \times\left[46080 \tilde{\alpha}^{4}(d-1)^{2} d^{2}\left(d^{2}-d-2\right)\right. \\
& +1920 \tilde{\alpha}^{3} \tilde{\beta} d\left(d^{6}-3 d^{5}+85 d^{4}-285 d^{3}\right.
\end{aligned}
$$

$$
\begin{aligned}
& \left.+106 d^{2}+288 d-192\right) \\
& +16 \tilde{\alpha}^{2} \tilde{\beta}^{2}\left(5 d^{8}-92 d^{7}+917 d^{6}-1886 d^{5}+7964 d^{4}\right. \\
& \left.-49268 d^{3}+42360 d^{2}+25920 d-23040\right) \\
& +8 \tilde{\alpha} \tilde{\beta}^{3} d\left(5 d^{7}-132 d^{6}+1235 d^{5}-4716 d^{4}\right. \\
& \left.+10988 d^{3}-28800 d^{2}-14880 d+46080\right) \\
& +\tilde{\beta}^{4}\left(5 d^{8}-142 d^{7}+1288 d^{6}-4628 d^{5}+7560 d^{4}\right. \\
& \left.\left.-19200 d^{3}-17280 d^{2}-46080 d+92160\right)\right] .
\end{aligned}
$$

In the EH limit we have

$$
\begin{aligned}
A_{1} & =\frac{4(d-3)}{(4 \pi)^{d / 2-1} \Gamma(d / 2)}, \\
B_{1} & =\frac{\left(d^{3}-15 d^{2}+24 d-72\right)}{3 d(4 \pi)^{d / 2-1} \Gamma(d / 2)}, \\
C_{1} & =\frac{5 d^{5}-147 d^{4}+1240 d^{3}-3612 d^{2}+2880 d-17280}{1440 d^{2}(4 \pi)^{d / 2} \Gamma(d / 2)} \\
D_{1} & =\frac{d^{3}-35 d^{2}+606 d-1080}{720(4 \pi)^{d / 2} \Gamma(d / 2)} .
\end{aligned}
$$

We note that the formulas for $B_{1}$ and $C_{1}+\frac{2}{d(d-1)} D_{1}$ do not agree with Eq. (5.7) in I. This is due to the different choice of cutoff (a function of the Bochner Laplacian in I and of the Lichnerowicz Laplacians here). Specializing to $d=4$, the formula for $C_{1}+\frac{2}{d(d-1)} D_{1}$ agrees with (5.6) in I but $B_{1}$ does not. This is a signal of the lack of universality of this coefficient.

\section{Conformal gravity in $d=4$}

Independently of the choice of gauge and parametrization, the effective action for conformal gravity is given formally by (3.10). This leads to the following flow for the effective action

$\dot{\Gamma}_{k}=\frac{1}{2} \operatorname{Tr}\left(\frac{\dot{\Delta}_{k}^{(2)}}{\Delta_{k}^{(2)}}\right)-\frac{1}{2} \operatorname{Tr}\left(\frac{\dot{\Delta}_{g h, k}^{(1)}}{\Delta_{g h, k}^{(1)}}\right)-\frac{1}{2} \operatorname{Tr}\left(\frac{\dot{\Delta}_{g h, k}^{(0)}}{\Delta_{g h, k}^{(0)}}\right)$,

where

$\Delta_{k}^{(2)}=\left(P_{k}\left(\Delta_{L 2}\right)-\frac{1}{2} \bar{R}\right)\left(P_{k}\left(\Delta_{L 2}\right)-\frac{1}{3} \bar{R}\right)$,

$\Delta_{k}^{(1)}=P_{k}\left(\Delta_{L 1}\right)-\frac{1}{2} \bar{R}$,

$\Delta_{k}^{(0)}=P_{k}\left(\Delta_{L 0}\right)-\frac{1}{3} \bar{R}$.

Proceeding as before we find

$$
\begin{aligned}
& A_{1}=\frac{3}{(4 \pi)^{2}}, \\
& B_{1}=\frac{41}{6(4 \pi)^{2}},
\end{aligned}
$$




$$
\begin{aligned}
& C_{1}=-\frac{199}{180(4 \pi)^{2}}, \\
& D_{1}=\frac{137}{60(4 \pi)^{2}} .
\end{aligned}
$$

We observe that the logarithmic divergences are given by

$$
\begin{gathered}
\frac{1}{(4 \pi)^{2}}\left[b_{4}\left(\Delta_{L 2}-\frac{1}{2} \bar{R}\right)+b_{4}\left(\Delta_{L 2}-\frac{1}{3} \bar{R}\right)\right. \\
\left.-b_{4}\left(\Delta_{L 1}-\frac{1}{2} \bar{R}\right)-b_{4}\left(\Delta_{L 2}-\frac{1}{2} \bar{R}\right)\right] .
\end{gathered}
$$

The coefficients $C_{1}$ and $D_{1}$ agree with previous calculations reported in $[37,38]$.

\section{Duality}

One of the main results of I was the existence of a discrete idempotent transformation leaving the functional measure, and the divergences, invariant. That invariance extends also to HDG, at least on an Einstein background. In any dimension and in any gauge we find that the divergences $A_{1}, B_{1}, C_{1}$ and $D_{1}$ have the duality symmetry:

$$
\begin{aligned}
& A_{1}(\omega, m)=A_{1}\left(1-\omega,-m-\frac{2}{d}\right), \\
& B_{1}(\omega, m)=B_{1}\left(1-\omega,-m-\frac{2}{d}\right), \\
& C_{1}(\omega, m)=B_{1}\left(1-\omega,-m-\frac{2}{d}\right), \\
& D_{1}(\omega, m)=C_{1}\left(1-\omega,-m-\frac{2}{d}\right) .
\end{aligned}
$$

This duality is only manifest if the coefficient $b$ enters in the gauge fixing (2.47) through the combination $\bar{b}=$ $b(1+d m)$. With other definitions of this coefficient a form of duality will still be present but it will have a much more complicated form. The calculations reported here indicate that this duality is not limited to Einstein gravity. It will be interesting to understand more generally under what circumstances this property holds.

A transformation can be an invariance of a quantum theory if it leaves invariant the action (and its expansion) and the functional measure. As discussed in the introduction, in our one-loop calculations we keep the functional measure fixed at (1.8) and the origin of the $\omega$ - and $m$-dependence of the results must lie in the form of the Hessians. One can indeed check explicitly that the Hessians given in Sects. 2.5 and 2.6 are duality-invariant.

Furthermore, it was observed in I that the functional measures

$$
\Pi_{x} d \gamma_{\mu \nu}(x)=\Pi_{x} d \hat{h}_{\mu \nu}(x)
$$

where $\gamma_{\mu \nu}$ and $\hat{h}_{\mu \nu}$ have weight $w$, and

$$
\Pi_{x} d \gamma^{\mu \nu}(x)=\Pi_{x} d \hat{h}^{\mu \nu}(x)
$$

where $\gamma^{\mu \nu}$ and $\hat{h}^{\mu \nu}$ have weight $w^{\prime}$, are equivalent. These weights are related by

$\frac{w^{\prime}}{2}=\frac{w}{2}+\frac{2}{d}, \quad$ which is the same as $m^{\prime}=-m-\frac{2}{d}$.

Thus, if we keep the same action, the functional integrals are equivalent. Again, these measures would give rise to different power-law divergence coefficients, but duality would still hold for each choice of measure.

Conversely, we could understand the invariance of the Hessian as follows. We have the functional integral

$\int\left[\Pi_{x} d \gamma_{\mu \nu}(x)\right] e^{-S\left[g_{\mu \nu}\right]}$,

where $\gamma_{\mu \nu}$ is related to the metric by [1]

$\gamma_{\mu \nu}=g_{\mu \nu}(\operatorname{det} g)^{w / 2}$.

Solving (6.4) for $g_{\mu \nu}$ and substituting it into (6.3), we have

$\int\left[\Pi_{x} d \gamma_{\mu \nu}(x)\right] e^{-S\left[\gamma_{\mu \nu}(\operatorname{det} \gamma)^{m}\right]}$,

with $m=-\frac{w / 2}{1+d w / 2}$. Simply rewriting $\gamma_{\mu \nu}$ as $g_{\mu \nu}$, we are lead to

$\int\left[\Pi_{x} d g_{\mu \nu}(x)\right] e^{-S\left[g_{\mu \nu}(\operatorname{det} g)^{m}\right]}$.

This is a functional integral with fixed quantum field, but the metric in the action is transformed. Viewed this way, the invariance should appear as an invariance of the action with the quantum field kept fixed. This is precisely the calculation we have given. The above discussion by the invariance of the measure suggests that duality is exact, but our calculation is done only at one loop. It would be interesting to check if the latter approach also gives the exact result.

The existence of the duality is, however, more general. If we used a functional measure that contains the determinant of a "De Witt" metric in functional space, the measure itself would be invariant under arbitrary field redefinitions [39]. In particular, it would be independent of $m$ and of the choices $\omega=0,1 / 2,1$. Such a measure would give rise to powerlaw divergence coefficients from the ones reported here, but duality would again appear because it is an invariance of the Hessian.

\section{Concluding remarks}

In this paper we have extended the analysis of I [1] from Einstein-Hilbert gravity to higher-derivative gravity containing the squares of the Ricci scalar and Ricci tensor. In 
four dimensions the analysis is essentially complete, because the remaining independent invariant is a total derivative. In higher dimensions this is not so. The analysis was also limited to Einstein backgrounds, which nearly solve the equations of motion, but also in this way is more general than the analysis in I, which was limited to maximally symmetric backgrounds.

We have obtained formulas for the one-loop divergences up to quadratic terms in the curvature. These are all the divergences that arise at one loop in $d=4$. The method we have used is a one-loop approximation of the single-field approximation of the exact RG equation for gravity, as first derived in [31] for the EH-case and then extended to HDG in [24,3436 ], to $3 d$ topologically massive gravity in [40], and beyond the one-loop approximation in [41-44].

The coefficients are related to the beta functions for the couplings. Comparing the action (2.1) with our results (4.3), we find the beta functions for the dimensionless couplings (4.6) as

$\beta_{\tilde{G}}=(d-2) \tilde{G}+B_{1} \tilde{G}^{2}$,

$\beta_{\tilde{\Lambda}}=-2 \tilde{\Lambda}+B_{1} \tilde{\Lambda} \tilde{G}+\frac{A_{1}}{2} \tilde{G}$,

$\beta_{\tilde{\alpha}+\frac{1}{d} \tilde{\beta}}=-(d-4)\left(\tilde{\alpha}+\frac{1}{d} \tilde{\beta}\right)+C_{1}$.

As already remarked, working on an Einstein space prevents us from disentangling the beta functions of $\tilde{\alpha}$ and $\tilde{\beta}$. On the other hand, even though we did not write a term $\gamma R_{\mu \nu \rho \sigma} R^{\mu \nu \rho \sigma}$ in the action, the divergence $D_{1}$ gives rise to a beta function

$\beta_{\tilde{\gamma}}=-(d-4) \tilde{\gamma}+D_{1}$.

The signs of the coefficients imply that $\alpha, \beta, \gamma$ are asymptotically free in $d=4$ (for a suitable range of parameters) [6-10] whereas $\tilde{G}$ goes in the UV to a fixed point $\tilde{G}=-(d-2) / B_{1}$. In order for this nontrivial fixed point to make sense, $B_{1}$ must be negative. In spite of the non-universality of the beta functions, many calculations indicate that $B_{1}$ is indeed negative for the linear split. For example, $B_{1}$ in (4.13) is negative for $m=\omega=0$ and a wide range of $a$ and $b$. With the exponential parametrization discussed in Sect. 4.6, $B_{1}$ in the EH limit given in (4.21) is negative for $3 \leq d \leq 13$. We refer the reader to the literature for a more detailed discussion.

The explicit coefficients of the divergences, in arbitrary dimension, gauge and parametrization, are too complicated to write, and we have exhibited only some special cases. The universal values agree with the literature. The divergences also agree, in $d=4$ and in the EH limit, with earlier calculations in general gauges $[29,45-48]$. For further discussions on the use of the exponential parametrization see [27,49-54].

Most of the general features noted in I persist in the theories considered here, as we have observed. In particular, one striking feature that we have checked in full generality is the existence of a "duality" symmetry under the change of parametrization (1.7), or

$\omega \rightarrow 1-\omega ; \quad m \rightarrow-m-\frac{2}{d}$.

As observed in I, this transformation preserves the dimension of the quantum field and is also an invariance of the functional measure. ${ }^{3}$ However, the parametrization dependence of the divergences is not due to the actual choice of the functional measure. Instead, it comes entirely from the different form of the Hessians in different parametrizations. Since these Hessians only differ by terms that are proportional to the equations of motion, the parametrization dependence, as well as the gauge dependence, goes away on shell.

Different choices of the ultralocal functional measure would alter the results for the power-law divergences coefficients. We refer to [57] and the references therein for a discussion of this point and to $[58,59]$ for more general results using Pauli-Villars regularization.

Whether the duality extends also to other classes of actions, to other backgrounds and to higher loops are all questions that we leave for further investigation. Also left for future work is the calculation of divergences in the unimodular case $m=-1 / d$, which contains in particular the unique self-dual theory $\omega=1 / 2, m=-1 / d$.

Acknowledgements This work was supported in part by the Grant-inAid for Scientific Research Fund of the JSPS (C) No. 16K05331. ADP is grateful to $\mathrm{CNPq}$ for financial support.

Open Access This article is distributed under the terms of the Creative Commons Attribution 4.0 International License (http://creativecomm ons.org/licenses/by/4.0/), which permits unrestricted use, distribution, and reproduction in any medium, provided you give appropriate credit to the original author(s) and the source, provide a link to the Creative Commons license, and indicate if changes were made.

Funded by SCOAP . $^{3}$

\section{Appendix A: $Q_{n}$ and heat kernel coefficients for Lich- nerowicz Laplacians}

Here we list the coefficients used in Sect. 4.1. The coefficients $Q_{n}$ are defined by

$Q_{n}=\frac{k^{2 n}}{\Gamma(n)} \int_{0}^{1} y^{n-1} d y=\frac{k^{2 n}}{\Gamma(n+1)}$.

The heat kernel coefficients for the Lichnerowicz Laplacians acting on spin- 0 , spin- 1 and spin- 2 fields on an Einstein manifold are

$b_{0}\left(\Delta_{L}^{(0)}\right)=1$,

$\overline{3}$ We recall that the pairs of measures proposed in $[55,56]$ are dual in this sense. 


$$
\begin{aligned}
b_{2}\left(\Delta_{L}^{(0)}\right)= & \frac{1}{6} R \\
b_{4}\left(\Delta_{L}^{(0)}\right)= & \frac{1}{180} R_{\mu \nu \rho \sigma} R^{\mu \nu \rho \sigma}+\frac{5 d-2}{360 d} R^{2}, \\
b_{0}\left(\Delta_{L}^{(1)}\right)= & d-1 \\
b_{2}\left(\Delta_{L}^{(1)}\right)= & \frac{d-7}{6} R \\
b_{4}\left(\Delta_{L}^{(1)}\right)= & \frac{d-16}{180} R_{\mu \nu \rho \sigma} R^{\mu \nu \rho \sigma}+\frac{5 d^{2}-67 d+182}{360 d} R^{2}, \\
b_{0}\left(\Delta_{L}^{(2)}\right)= & \frac{(d+1)(d-2)}{2}, \\
b_{2}\left(\Delta_{L}^{(2)}\right)= & \frac{d^{2}-13 d-14}{12} R, \\
b_{4}\left(\Delta_{L}^{(2)}\right)= & \frac{d^{2}-31 d+508}{360} R_{\mu \nu \rho \sigma} R^{\mu \nu \rho \sigma} \\
& +\frac{5 d^{3}-127 d^{2}+592 d+1804}{720 d} R^{2} .
\end{aligned}
$$

These formulas can be obtained by the methods described for example in Appendix B of [60]. Here we do not take into account isolated modes that do not contribute to the fluctuation $h_{\mu \nu}$. Thus these formulas hold in the case when the manifold has no Killing or conformal Killing vectors, or else if the manifold is noncompact and has a continuous spectrum, so that the spurious isolated modes are of measure zero.

In order to get the heat kernel coefficients of shifted Lichnerowicz Laplacians $\Delta+a R$, one can use

$$
\begin{aligned}
& b_{0}(\Delta+a R)=b_{0}(\Delta) \\
& b_{2}(\Delta+a R)=b_{2}(\Delta)-a R b_{0}(\Delta), \\
& b_{4}(\Delta+a R)=b_{4}(\Delta)-a R b_{2}(\Delta)+\frac{1}{2} a^{2} R^{2} b_{0}(\Delta) .
\end{aligned}
$$

\section{References}

1. N. Ohta, R. Percacci, A.D. Pereira, Gauges and functional measures in quantum gravity I: Einstein theory. JHEP 1606, 115 (2016). arXiv:1605.00454 [hep-th]

2. L. Modesto, Super-renormalizable quantum gravity. Phys. Rev. D 86, 044005 (2012). arXiv:1107.2403 [hep-th]

3. T. Biswas, E. Gerwick, T. Koivisto, A. Mazumdar, Towards singularity and ghost free theories of gravity. Phys. Rev. Lett. 108, 031101 (2012). arXiv:1110.5249 [gr-qc]

4. L. Modesto, L. Rachwal, Super-renormalizable and finite gravitational theories. Nucl. Phys. B 889, 228 (2014). arXiv:1407.8036 [hep-th]

5. K.S. Stelle, Renormalization of higher derivative quantum gravity. Phys. Rev. D 16, 953 (1977)

6. J. Julve, M. Tonin, Quantum gravity with higher derivative terms. Nuovo Cim. B 46, 137 (1978)

7. E.S. Fradkin, A.A. Tseytlin, Renormalizable asymptotically free quantum theory of gravity. Phys. Lett. B 104, 377 (1981)

8. E.S. Fradkin, A.A. Tseytlin, Renormalizable asymptotically free quantum theory of gravity. Nucl. Phys. B 201, 469 (1982)
9. I.G. Avramidi, A.O. Barvinsky, Asymptotic freedom in higher derivative quantum gravity. Phys. Lett. B 159, 269 (1985)

10. I.G. Avramidi, Covariant methods for the calculation of the effective action in quantum field theory and investigation of higher derivative quantum gravity. $\mathrm{PhD}$ thesis, Moscow State University (1986); UDK 530.12:531.51. arXiv:hep-th/9510140

11. A. Salam, J.A. Strathdee, Remarks on high-energy stability and renormalizability of gravity theory. Phys. Rev. D 18, 4480 (1978)

12. R. Floreanini, R. Percacci, The renormalization group flow of the Dilaton potential. Phys. Rev. D 52, 896 (1995). arXiv:hep-th/9412181

13. A. Bonanno, M. Reuter, Modulated ground state of gravity theories with stabilized conformal factor. Phys. Rev. D 87, 084019 (2013). arXiv:1302.2928 [hep-th]

14. I.L. Shapiro, Counting ghosts in the ghost-free non-local gravity. Phys. Lett. B 744, 67 (2015). arXiv:1502.00106 [hep-th]

15. E. Tomboulis, $1 / \mathrm{N}$ expansion and renormalization in quantum gravity. Phys. Lett. B 70, 361 (1977)

16. E. Tomboulis, Renormalizability and asymptotic freedom In quantum gravity. Phys. Lett. B 97, 77 (1980)

17. E.T. Tomboulis, Unitarity in higher derivative quantum gravity. Phys. Rev. Lett. 52, 1173 (1984)

18. P.D. Mannheim, Solution to the ghost problem in fourth order derivative theories. Found. Phys. 37, 532 (2007). arXiv:hep-th/0608154

19. S. Mukohyama, J.P. Uzan, From configuration to dynamics: emergence of Lorentz signature in classical field theory. Phys. Rev. D 87, 065020 (2013). arXiv:1301.1361 [hep-th]

20. A. Salvio, A. Strumia, Agravity. JHEP 1406, 080 (2014). arXiv:1403.4226 [hep-ph]

21. M.B. Einhorn, D.R.T. Jones, Naturalness and dimensional transmutation in classically scale-invariant gravity. JHEP 1503, 047 (2015). arXiv: 1410.8513 [hep-th]

22. L. Alvarez-Gaume, A. Kehagias, C. Kounnas, D. Lüst, A. Riotto, Aspects of quadratic gravity. Fortsch. Phys. 64, 176 (2016). arXiv: 1505.07657 [hep-th]

23. K.A. Kazakov, P.I. Pronin, Gauge and parametrization dependence in higher derivative quantum gravity. Phys. Rev. D 59, 064012 (1999). arXiv:hep-th/9806023

24. N. Ohta, R. Percacci, Higher derivative gravity and asymptotic safety in diverse dimensions. Class. Quant. Grav. 31, 015024 (2014). arXiv:1308.3398 [hep-th]

25. H. Hata, T. Kugo, N. Ohta, Skew symmetric tensor gauge field theory dynamically realized in QCD U(1) channel. Nucl. Phys. B 178, 527 (1981)

26. T. Kugo, S. Uehara, General procedure of gauge fixing based on BRS invariance principle. Nucl. Phys. B 197, 378 (1982)

27. R. Percacci, G.P. Vacca, Search of scaling solutions in scalar-tensor gravity. Phys. Rev. D 92, 061501 (2015). arXiv:1501.00888 [hepth]

28. R. Percacci, M.J. Perry, C.N. Pope, E. Sezgin, Beta functions of topologically massive supergravity. JHEP 1403, 083 (2014). arXiv:1302.0868 [hep-th]

29. E.S. Fradkin, A.A. Tseytlin, One loop effective potential in gauged O(4) supergravity. Nucl. Phys. B 234, 472 (1984)

30. S.M. Christensen, M.J. Duff, Quantizing gravity with a cosmological constant. Nucl. Phys. B 170, 480 (1980)

31. M. Reuter, Nonperturbative evolution equation for quantum gravity. Phys. Rev. D 57, 971 (1998). arXiv:hep-th/9605030

32. D. Dou, R. Percacci, The running gravitational couplings. Class. Quant. Grav. 15, 3449 (1998). arXiv:hep-th/9707239

33. G. de Berredo-Peixoto, I.L. Shapiro, Higher derivative quantum gravity with Gauss-Bonnet term. Phys. Rev. D 71, 064005 (2005). arXiv:hep-th/0412249

34. A. Codello, R. Percacci, Fixed points of higher derivative gravity. Phys. Rev. Lett. 97, 221301 (2006). arXiv:hep-th/0607128 
35. M. Niedermaier, Gravitational fixed points from perturbation theory. Phys. Rev. Lett. 103, 101303 (2009)

36. M. Niedermaier, Gravitational fixed points and asymptotic safety from perturbation theory. Nucl. Phys. B 833, 226 (2010)

37. G. de Berredo-Peixoto, I.L. Shapiro, Conformal quantum gravity with the Gauss-Bonnet term. Phys. Rev. D 70, 044024 (2004). arXiv:hep-th/0307030

38. Y. Pang, One-loop divergences in 6D conformal gravity. Phys. Rev. D 86, 084039 (2012). arXiv:1208.0877 [hep-th]

39. E. Mottola, Functional integration over geometries. J. Math. Phys. 36, 2470 (1995). arXiv:hep-th/9502109

40. R. Percacci, E. Sezgin, One loop beta functions in topologically massive gravity. Class. Quant. Grav. 27, 155009 (2010). arXiv:1002.2640 [hep-th]

41. O. Lauscher, M. Reuter, Flow equation of quantum Einstein gravity in a higher derivative truncation. Phys. Rev. D 66, 025026 (2002). arXiv:hep-th/0205062

42. D. Benedetti, P.F. Machado, F. Saueressig, Asymptotic safety in higher-derivative gravity. Mod. Phys. Lett. A 24, 2233 (2009). arXiv:0901.2984 [hep-th]

43. D. Benedetti, P.F. Machado, F. Saueressig, Taming perturbative divergences in asymptotically safe gravity. Nucl. Phys. B 824, 168 (2010). arXiv:0902.4630 [hep-th]

44. K. Groh, S. Rechenberger, F. Saueressig, D. Zanusso, Higher derivative gravity from the universal renormalization group machine. PoS EPS HEP2011, 124 (2011). arXiv:1111.1743 [hepth]

45. R.E. Kallosh, O.V. Tarasov, I.V. Tyutin, One loop finiteness of quantum gravity off mass shell. Nucl. Phys. B 137, 145 (1978)

46. M.Y. Kalmykov, Gauge and parametrization dependencies of the one loop counterterms in the Einstein gravity. Class. Quant. Grav. 12, 1401 (1995). arXiv:hep-th/9502152

47. M.Y. Kalmykov, K.A. Kazakov, P.I. Pronin, K.V. Stepanyantz, Detailed analysis of the dependence of the one loop counterterms on the gauge and parametrization in the Einstein gravity with the cosmological constant. Class. Quant. Grav. 15, 3777 (1998). arXiv:hep-th/9809169
48. H. Gies, B. Knorr, S. Lippoldt, Generalized parametrization dependence in quantum gravity. Phys. Rev. D 92, 084020 (2015). arXiv: 1507.08859 [hep-th]

49. A. Nink, Field parametrization dependence in asymptotically safe quantum gravity. Phys. Rev. D 91, 044030 (2015). arXiv:1410.7816 [hep-th]

50. M. Demmel, A. Nink, Phys. Rev. D 92, 104013 (2015). arXiv: 1506.03809 [gr-qc]

51. N. Ohta, R. Percacci, Ultraviolet fixed points in conformal gravity and general quadratic theories. Class. Quant. Grav. 33, 035001 (2016). arXiv:1506.05526 [hep-th]

52. N. Ohta, R. Percacci, G.P. Vacca, Flow equation for $f(R)$ gravity and some of its exact solutions. Phys. Rev. D 92, 061501 (2015). arXiv: 1507.00968 [hep-th]

53. N. Ohta, R. Percacci, G.P. Vacca, Renormalization group equation and scaling solutions for $\mathrm{f}(\mathrm{R})$ gravity in exponential parametrization. Eur. Phys. J. C 76, 46 (2016). arXiv: 1511.09393 [hep-th]

54. K. Falls, N. Ohta, Renormalization group equation for $f(R)$ gravity on hyperbolic spaces. Phys. Rev. D 94, 084005 (2016). arXiv:1607.08460 [hep-th]

55. K. Fujikawa, Path integral measure for gravitational interactions. Nucl. Phys. B 226, 437 (1983)

56. K. Fujikawa, O. Yasuda, Path integral for gravity and supergravity. Nucl. Phys. B 245, 436 (1984)

57. D.J. Toms, The functional measure for quantum field theory in curved space-time. Phys. Rev. D 35, 3796 (1987)

58. D. Anselmi, Functional integration measure in quantum gravity. Phys. Rev. D 45, 4473 (1992)

59. D. Anselmi, On delta $(0)$ divergences and the functional integration measure. Phys. Rev. D 48, 680 (1993)

60. A. Codello, R. Percacci, C. Rahmede, Investigating the ultraviolet properties of gravity with a Wilsonian renormalization group equation. Annals Phys. 324, 414 (2009). arXiv:0805.2909 [hep-th] 